\title{
Corela
}

Cognition, représentation, langage

HS-6 | 2007

Cognition, discours, contextes

\section{La cognition humaine saisie par le langage : de la sémantique cognitive au médiationnisme}

\section{Vincent Nyckees}

\section{CpenEdition}

\section{Journals}

Édition électronique

URL : http://journals.openedition.org/corela/1538

DOI : $10.4000 /$ corela. 1538

ISSN : 1638-573X

\section{Éditeur}

Cercle linguistique du Centre et de I'Ouest - CerLICO

\section{Référence électronique}

Vincent Nyckees, « La cognition humaine saisie par le langage : de la sémantique cognitive au médiationnisme », Corela [En ligne], HS-6 | 2007, mis en ligne le 01 novembre 2007, consulté le 01 mai 2019. URL : http://journals.openedition.org/corela/1538; DOI : 10.4000/corela.1538

Ce document a été généré automatiquement le 1 mai 2019.

\section{(c) (i) (2)(2)}

Corela - cognition, représentation, langage est mis à disposition selon les termes de la licence Creative Commons Attribution - Pas d'Utilisation Commerciale - Partage dans les Mêmes Conditions 4.0 International. 


\title{
La cognition humaine saisie par le langage : de la sémantique cognitive au médiationnisme
}

\author{
Vincent Nyckees
}

1 Si l'on en croit une opinion répandue chez les partisans de la sémantique cognitive, la seule « alternative » à l'objectivisme ${ }^{1}$ - et, par conséquent, la seule théorie cognitive possible serait un expérientialisme de type mentaliste, celui-là même qu'ils défendent et illustrent dans leurs travaux. D'où il résulterait que toute contestation de la sémantique cognitive signerait un retour à l'objectivisme tant critiqué. Cette conviction repose manifestement sur le raisonnement suivant :

- les significations linguistiques ne peuvent être analysées adéquatement dans le cadre objectiviste parce qu'elles requièrent la compréhension humaine ;

- elles sont par conséquent de nature conceptuelle et doivent être analysées intégralement par référence à des mécanismes mentaux.

2 Nous voudrions montrer dans cet article que cette inférence ne s'impose pas, que le rejet de l'objectivisme ne nous réduit pas au mentalisme et qu'une autre linguistique cognitive est possible, si l'on entend par linguistique cognitive une approche: $i$ ) qui estime que toute signification est nécessairement relative à la compréhension humaine ; ii) qui se fixe pour objectif d'éclairer aussi précisément que possible les processus selon lesquels les sujets humains confèrent du sens aux unités, aux structures et aux énoncés linguistiques.

Nous nous proposons deux objectifs complémentaires.

3 Nous voulons d'abord faire état des très sérieuses objections que soulèvent selon nous les thèses de la sémantique cognitive actuelle, du fait, essentiellement, de leur mentalisme, autrement dit de la réduction univoque des significations linguistiques qu'elles proposent à des représentations mentales préexistantes. Nous prendrons essentiellement pour référence de notre analyse la grande construction théorique, véritable "système sémantique » à quatre mains, élaborée dans une étonnante synergie par Lakoff et Johnson dans leurs ouvrages respectifs de 1987, ouvrages qui présentent, bien plus que leurs 
autres travaux, les bases d'une théorie complète du langage et de la signification et qui proposent, pour l'illustrer, des études de cas particulièrement importantes et stimulantes 2 .

4 Nous entendons également démontrer la supériorité, sur ces mêmes points, dans l'ordre de la théorie sémantique comme dans le traitement des données empiriques, d'une approche cognitive d'un type nouveau, que nous qualifions de médiationniste ( $c f$. Nyckees 2003) prenant en compte le rôle déterminant du langage - et, plus généralement, des grands vecteurs de l'activité humaine : signes, textes, artefacts... - dans l'évolution de la pensée propre à notre espèce, tant au plan individuel (via, essentiellement, l'acquisition des significations et les transformations de la compétence individuelle) qu'au plan collectif (à travers, tout particulièrement, l'histoire des langues et des significations). Pour le médiationnisme, en effet, la spécificité de la cognition humaine, considérée dans son fonctionnement, par rapport à la cognition animale procède pour l'essentiel de l'usage de systèmes de signes (et d'abord de systèmes linguistiques) ${ }^{3}$. Non pas que ces systèmes doivent être tenus pour les produits d'une génération spontanée: leur émergence a présupposé, selon toute vraisemblance, certaines dispositions spécifiques nouvelles dont les spécialistes peuvent tenter de reconstituer la genèse, comme celles que résume le concept d'intentionnalité partagée forgé par Tomasello et al. (2005). Il s'agit de souligner en revanche que, sans le développement (multimillénaire) du langage dans notre espèce, la propension des individus humains à une véritable activité conjointe avec leurs semblables n'aurait pu suffire à redéfinir aussi radicalement leur monde initial. Le langage a manifestement révolutionné l'existence humaine, en affectant aussi bien le développement cognitif individuel que les conditions collectives, historiques, de notre activité et de notre pensée ( $c f$. infra, 4.).

5 La perspective médiationniste que nous préconisons participe d'un paradigme externaliste très productif aujourd'hui en sciences cognitives, philosophie et sciences sociales, affirmant le caractère socio-culturel et « historique » de la cognition dans notre espèce et le rôle déterminant qu'y jouent les signes et/ou les artefacts (courants de la cognition située ou distribuée, nouvelles philosophies de la technique de Stiegler, Derrida, Havelange, relayant à leur manière la pensée fondatrice d'un Leroi-Gourhan, etc.). L'approche médiationniste prend donc acte de la "constitutivité » culturelle, technique et, au premier chef, sémiotique de la cognition humaine. Elle se reconnaît des ancêtres et des aînés dans une longue lignée de penseurs et de chercheurs dont la figure la plus représentative au XXème siècle fut certainement Lev Vygotski, pour ne rien dire de Schaff, Bruner, Tomasello, voire Changeux ou Edelman. Dans le champ linguistique, malheureusement, et malgré l'écho indéniable qu'un certain esprit médiationniste a toujours rencontré et qu'il rencontre encore chez divers auteurs (cf. Bréal, Saussure, Benveniste, Martinet et, plus près de nous, Hagège, Auroux, Rastier, Bronckart ou Paveau), cette option théorique est restée dans les limbes et, faute d'occasion, n'a pas débouché à ce jour sur une théorie explicite du langage et de la signification. C'est dans le sens de cette élaboration théorique que nos propres travaux de sémantique ne cessent de nous pousser depuis de nombreuses années ${ }^{4}$, depuis, notamment, que nous avons pu constater l'incapacité d'une sémantique cognitive d'inspiration mentaliste à résoudre les problèmes sémantiques qu'elle mettait elle-même en avant ${ }^{5}$. Nous avons en outre acquis peu à peu la certitude qu'en prenant mieux en compte la dimension historique des langues et la variabilité synchronique ${ }^{6}$ des compétences linguistiques, une approche 
médiationniste permettait d'atteindre un degré encore inédit d'adéquation descriptive et de puissance explicative dans l'analyse des faits de langue.

6 Après une présentation détaillée et aussi impartiale que possible des principales thèses de la sémantique cognitive actuelle ${ }^{7}$ (1.), nous procéderons à leur évaluation méthodique ainsi qu'à l'examen des principaux concepts explicatifs qu'elle met en œuvre ${ }^{8}$ et nous exposerons systématiquement sur tous ces points les contre-analyses et propositions qu'inspire une approche cognitive de type médiationniste (2. et 3.).

7 Nous nous concentrerons particulièrement sur les deux questions étroitement liées de la catégorisation et de la structuration des catégories conceptuelles (pour employer les termes de la sémantique cognitive) parce qu'elles engagent plus que d'autres la question de la nature de la signification linguistique et qu'elles occupent une place considérable dans les analyses et les réflexions de nos auteurs comme dans les travaux de la sémantique actuelle. Nous formulerons ainsi les principes d'un modèle médiationniste de la symbolisation linguistique et proposerons une réinterprétation non objectiviste du modèle des conditions nécessaires et suffisantes, échappant - selon nous - tant aux difficultés soulevées par l'interprétation objectiviste de ce modèle qu'aux défauts des approches mentalistes de la catégorisation. Nous spécifierons chemin faisant la théorie (" casuelle ») de la signification solidaire de notre modèle des CNS révisé. Nous réanalyserons ensuite plusieurs cas réputés rebelles aux CNS traditionnelles.

8 Enfin (4.), nous tenterons de démontrer la nécessité impérieuse, pour la théorie du langage comme pour les études cognitives, de prendre en compte la constitutivité sémiotique de notre cognition et de se recentrer sur la double historicité, individuelle et collective, du langage humain et de la cognition humaine.

\section{Le monde de signification de la sémantique cognitive}

\subsection{Le mentalisme}

9 La sémantique cognitive se définit avant tout par son mentalisme. Elle postule que le langage reçoit sa signification des structures de notre système conceptuel $l^{9}$ et que les propriétés du langage s'expliquent à peu près intégralement par les propriétés de la cognition humaine, lesquelles, selon nos auteurs, sont elles-mêmes fonction de la condition biologique de l'homme, invariante dans ses grandes lignes à travers l'espace et le temps, ainsi que des contraintes de l'environnement. C'est pourquoi ${ }^{10}$ Lakoff peut annoncer, p. 67, qu'il étudiera l'appareil cognitif utilisé dans la catégorisation à partir des données offertes par les langues en vertu de l'hypothèse de travail selon laquelle ${ }^{11}$ "le langage fait vraiment usage de mécanismes cognitifs généraux, au moins des mécanismes de catégorisation ».

10 Ce mentalisme est, à l'époque de Women..., essentiellement innéiste, à la fois nativiste et expérientaliste selon les propres termes de Lakoff ${ }^{12}$ (XV, 146 et chap. 17). 


\subsection{Un modèle général de la signifiance : l'ancrage dans l'expérience corporelle préconceptuelle et le rôle de « l'imagination »}

11 La théorie de la signification implique pour Lakoff comme pour Johnson une théorie de la compréhension. Il s'agit d'expliquer comment les formes linguistiques prennent leur signification pour les sujets cognitifs (et, plus généralement, tout ce qui a du sens dans l'acception la plus large de ce mot - car la sémantique cognitive rejette toute différence fondamentale de nature entre les types de signification). Or, dans ce cadre mentaliste, l'invocation de la compréhension humaine conduit à placer toute signification sous la dépendance d'une théorie de la conceptualisation : « Le langage est rendu signifiant parce qu'il est directement relié à la pensée signifiante et dépend de la nature de la pensée » (Lakoff : 291); quant à notre pensée et à notre raison, elles sont elles-mêmes rendues signifiantes par leurs connexions avec notre fonctionnement corporel préconceptuel ( ibid.). Ainsi, pour Lakoff (372), notre pensée et notre raison mettent en œuvre des «structures symboliques» qui signifient (s'ancrent dans notre expérience) soit directement (concepts de niveau de base et schèmes d'images), soit indirectement (en mettant en œuvre nos capacités imaginatives).

\subsubsection{Les structures directement signifiantes}

La sémantique cognitive a impérativement besoin de structures directement signifiantes pour "brancher " directement la conceptualisation humaine sur la perception et la motricité, en vertu du postulat mentaliste qui fait du langage une simple expression de la pensée $^{13}$ : "les concepts de niveau de base et de schèmes d'images sont compris directement dans les termes de l'expérience physique » (Lakoff : 282).

\subsubsection{Les concepts du niveau de base}

13 Ils « reflét[ent] la structure de notre expérience perceptivo-motrice et notre capacité à former des images mentales» (Lakoff: 372$)^{14}$. C'est au niveau de base que s'opère la catégorisation première de notre monde physique (sensori-moteur) et que sont acquises les images mentales (les « rich images » de Johnson). Ce niveau de base est défini comme le niveau par excellence auquel l'homme interagit avec son environnement: "C'est à ce niveau d'expérience physique que nous distinguons correctement les tigres des éléphants, les chaises des tables, les roses des jonquilles, les asperges des brocolis, le cuivre du laiton, etc. » (Lakoff : $269 ;$;f. aussi Johnson : 208-209).

On retrouve avec le niveau de base les images mentales qui servaient d'entrée à la conceptualisation chez Locke.

\subsubsection{Les schèmes d'images}

Les schèmes d'images sont des "patterns dynamiques" (Johnson: 2) que nous développons «pour interagir efficacement [for interacting forcefully] avec notre environnement " (Johnson: 13 - on notera le finalisme de la formule). "Un schème d'image (...) fonctionne à peu près comme une structure abstraite d'image et relie ensemble de cette façon une grande série d'expériences différentes qui manifestent la même structure récurrente. " (Johnson : 2). Ainsi, le schème d'image du CHEMIN [FROM- 
TO or PATH schema] « consiste en trois éléments (un point-source A, un point-cible B, un vecteur traçant un chemin entre eux) et une relation (caractérisée comme une force se déplaçant de $A$ vers $B$ ). Ce schème FROM-TO est une structure récurrente qui se manifeste dans un certain nombre d'événements apparemment différents tels que : a) se déplacer à pied d'un endroit à un autre, b) lancer à votre sœur une balle de base-ball c) donner un coup de poing à votre frère, d) offrir un cadeau à votre mère, e) ou, s'agissant de la glace, passer du solide au liquide. Pour chacun de ces cas très différents nous avons le même schème avec les mêmes composants et relations élémentaires. » (Johnson : 28)

Les schèmes d'images jouent un rôle essentiel dans notre conceptualisation et nos raisonnements parce qu'ils présentent tout à la fois un degré d'abstraction intermédiaire qui les rend éminemment transposables sans les démotiver pour autant aux yeux des sujets (grâce à leur récurrence dans l'expérience préconceptuelle) et une flexibilité élevée qui leur permet de reconfigurer quantité de domaines conceptuels, comme nous le verrons plus loin.

17 Ainsi, pour Lakoff, comme pour Johnson, l'essentiel de la « conceptualisation » se joue au niveau préconceptuel, donc $a$ fortiori prélinguistique.

\subsubsection{Les structures indirectement signifiantes}

18 Elles sont «élaborées par les capacités imaginatives (spécialement la métaphore et la métonymie) " (Lakoff : 372). La conceptualisation humaine prend donc pour entrée les concepts de niveau de base et les schèmes d'images et les utilise pour motiver des concepts qui s'accordent avec ces structures préconceptuelles » (Lakoff : 303). Ainsi, c'est la perception telle que la rend possible l'équipement biologique (inné) de l'homme - non modifiée par le langage - qui sert d'entrée à la totalité des concepts et schèmes de base, lesquels pourront donner lieu à des concepts dérivés par le jeu des projections métaphoriques ou métonymiques (elles-mêmes pensées par Lakoff et Johnson comme pouvant se produire indépendamment de toute expression linguistique). On dispose ainsi de tout ce qui est nécessaire à la cognition humaine sans avoir besoin, à aucun moment, d'en passer par le langage.

19 Signalons d'emblée trois particularités importantes de l'analyse que Johnson nous propose de la métaphore, et qui pourraient tout aussi bien s'appliquer à la métonymie s'il lui accordait la même attention.

Johnson, en premier lieu, dissocie nettement métaphore et langage :

...Je n'utiliserai pas «métaphore» dans le sens traditionnel comme désignant simplement une figure de discours; je l'identifierai plutôt comme une structure indispensable et omniprésente de la compréhension humaine à l'aide de laquelle nous comprenons notre monde de manière figurée » (Johnson: XX). A propos de la métaphore LE TEMPS, C'EST DE L'ARGENT, Johnson reconnaît ainsi (104) qu'il utilise le terme de métaphore «en un sens étendu métaphoriquement et non seulement comme une connexion propositionnelle de deux domaines d'expérience déjà déterminés, aux contours nettement définis.

20 Soulignons ensuite que les métaphores retenues comme exemples par Johnson (ou par Lakoff) ne sont jamais analysées en tant qu'énonciations singulières, alors même qu'elles nous sont présentées sous forme d'énoncés, mais sont systématiquement traitées au contraire comme de simples occurrences de modèles métaphoriques généraux inscrits dans notre système conceptuel à titre de patterns universels ou de stéréotypes culturels (LE TEMPS, C'EST DE L'ARGENT, L'APPARENCE PHYSIQUE EST UNE FORCE PHYSIQUE). 
L'analyse ramène donc invariablement la métaphore à un niveau prélinguistique et prédiscursif.

On notera enfin que Johnson considère les projections métaphoriques comme une activité largement inconsciente, voire automatique, arguant que « la plus grande partie de notre compréhension est, en fait active, à un niveau qui échappe le plus souvent à notre conscience réflexive [of which we are seldom reflectively aware] » (Johnson : 82).

\subsubsection{Les projections métaphoriques}

Dans les domaines où il n'existe pas de structure préconceptuelle clairement discernable dans notre expérience, nous importons une telle structure à l'aide de la métaphore. La métaphore nous fournit un moyen d'appréhender des domaines d'expérience qui n'ont pas de structure préconceptuelle propre. Un grand nombre de nos domaines d'expérience sont de ce type (Lakoff : 303).

Soit, par exemple, la « métaphore » LE PLUS EST EN HAUT [MORE IS UP] (à supposer que l'on s'accorde avec Lakoff, et tous les sémanticiens cognitivistes qui ont repris cet exemple, pour parler en pareil cas de métaphore, $c f$., infra, pour une critique). Lakoff nous explique que cette « métaphore » est motivée, dans son ensemble comme dans ses détails, par une corrélation structurelle dans notre expérience :

Chaque fois que nous ajoutons quelque chose d'une substance - disons, de l'eau dans un verre - le niveau s'élève. Quand nous ajoutons des objets sur une pile, le niveau monte. Retirez des objets sur la pile ou de l'eau dans le verre et le niveau baisse. La corrélation est irrésistible :

PLUS est corrélé avec EN HAUT, MOINS est corrélé avec EN BAS. (Lakoff : 276)

$\mathrm{Du}$ fait de cette corrélation extrêmement régulière, la VERTICALITÉ nous sert, selon Lakoff (277), de « domaine source approprié pour comprendre la QUANTITÉ ».

Nombre de nos significations et, par suite, de nos raisonnements seraient ainsi structurés par des projections métaphoriques.

\subsubsection{Les projections métonymiques}

"Certains modèles cognitifs sont métonymiques, nous dit Lakoff(153), en ce qu'ils permettent à une partie d'une catégorie (un membre ou une sous-catégorie) de représenter la catégorie dans son ensemble à certaines fins, ordinairement pour le raisonnement». On peut distinguer, selon l'auteur, deux types de projections métonymiques. Dans les cas les plus classiques, comme dans la métonymie banale du lieu pour l'institution qui l'occupe (la Maison Blanche pour la Présidence des Etats-Unis), la projection métonymique peut être systématiquement rapportée à tel ou tel principe général ( $c f$. Lakoff: $77 \mathrm{sq}$ ). Lakoff reconnaît d'ailleurs volontiers que ces principes généraux "ne sont pas les mêmes dans toutes les langues" (78). Dans d'autres cas en revanche (les catégories radiales), aucun principe général ne peut être mis en évidence, ce qui n'exclut pas que la relation soit motivée ( $c f$. infra). Lakoff (p. 90 ; et tout au long du chap. 5) nous invite à ranger parmi les modèles métonymiques :

- les «stéréotypes sociaux », tel celui qui fait considérer la sous-catégorie des mères au foyer comme plus représentative de la catégorie mère que celle des "mères actives " [working mothers] ;

- les « exemples typiques» (comme le merle et le moineau pour la catégorie oiseau); 
- les « cas idéaux » (le mari idéal) ;

- les « parangons » (une vraie Babe Ruth, la Cadillac des aspirateurs);

- les « générateurs ", membres centraux d'une catégorie qui en « engendrent » d'autres par application de règles générales (par ex. les nombres à un chiffre engendrant la catégorie entière des nombres entiers moyennant certains principes arithmétiques généraux) ;

- les «sous-modèles" (ainsi, dans la catégorie des nombres naturels, le sous-modèle des multiples de 10 qui nous permet communément d'arrondir un chiffre à la dizaine, voire à la centaine ou au millier supérieur) ;

- les « exemples saillants " («si votre meilleur ami est végétarien et que vous ne connaissez guère d'autres végétariens, vous serez enclin à développer des généralités sur les végétariens à partir du cas de votre ami », Lakoff : 89)

27 Ces différents types de "modèles métonymiques" sont, selon Lakoff, responsables de nombreux effets prototypiques, mais ils méritent surtout notre attention pour le rôle qu'ils jouent dans nos raisonnements en nous permettant d'extrapoler à des cas ou des domaines moins connus de nous (ou plus difficiles à appréhender) nos connaissances ou nos croyances relatives à des cas ou des domaines plus familiers.

\subsubsection{L'hypothèse de la spatialisation de la forme}

Schèmes d'images et projections métaphoriques et métonymiques acquièrent une puissance explicative décisive dans les travaux de Lakoff et de Johnson en opérant leur jonction à la faveur de l'hypothèse dite de la spatialisation de la forme. Cette articulation permet, selon Lakoff (282-283), de jeter les bases d'une théorie de la structure conceptuelle générale " apte à rendre compte de la structure abstraite de nos concepts. L'hypothèse de la spatialisation de la forme sur laquelle repose cette théorie stipule en effet que les schèmes d'images ne structurent pas seulement notre expérience de l'espace, mais qu'ils structurent nos concepts eux-mêmes, par projection sur la structure conceptuelle de la structure spatiale saisie par les schèmes d'images :

...Les schèmes d'images (qui structurent l'espace) sont projetés sur les configurations abstraites correspondantes (qui structurent les concepts). (Lakoff : 283)

Et Lakoff d'énumérer un certain nombre de structures abstraites fondamentales que nous comprendrions à l'aide de schèmes d'images :

- Les catégories (en général) sont comprises dans les termes des schèmes du CONTENANT.

- La structure hiérarchique est comprise dans les termes des schèmes PARTIE-TOUT et HAUT-BAS.

- La structure relationnelle est comprise dans les termes des schèmes de LIENS.

- La structure radiale des catégories est comprise dans les termes des schèmes CENTRE-PÉRIPHÉRIE... (283)

30 À la faveur de la spatialisation de la forme, les schèmes d'images joueraient également un rôle déterminant dans la genèse du raisonnement logique : "[Les schèmes d'images] comportent aussi une logique basique interne que nous croyons suffisante pour caractériser la raison humaine » (Lakoff : 372 ). On ne s'étonnera donc pas de voir Johnson (37-40) entreprendre de dériver toutes les lois du raisonnement logique du schème d'image du CONTENANT. Quant à Lakoff (379), il ne craint pas de considérer l'élaboration d'une « méthodologie permettant d'étudier la structure linguistique et conceptuelle dans 
ses moindres détails " comme une tâche essentielle de la linguistique cognitive - et comme l'un de ses apports majeurs aux autres branches des sciences cognitives.

\subsection{Un modèle pluriel de la structuration des catégories}

\subsubsection{Un traitement essentiellement négatif de la question de la catégorisation}

31 Si toutes nos connaissances dérivent directement ou indirectement de notre expérience préconceptuelle, il reste à déterminer comment nous les appliquons au monde qui nous entoure et comment nous les organisons en structurant nos concepts et/ou nos significations, ce qui, dans un cadre mentaliste - n'accordant au langage aucun rôle dans la conceptualisation -, revient à spécifier un modèle de la catégorisation et un modèle de la structuration de nos catégories conceptuelles. Il convient toutefois d'observer que, depuis qu'elle a renoncé à ce que Kleiber appellerait la « version standard du prototype » - c'està-dire, si l'on en croit Lakoff (43 sq et 136 sq), depuis fort longtemps -, la sémantique cognitive s'est beaucoup plus intéressée au second problème qu'au premier. Si, en effet, la réflexion sur la catégorisation présente encore dans ses travaux une actualité indéniable (aucun thème ne lui tient sans doute plus à cœur), l'objet qui lui est assigné s'est sensiblement déplacé ${ }^{15}$, le problème principal n'étant plus tant de tirer au clair les conditions selon lesquelles des occurrences particulières ou des faisceaux particuliers de percepts se trouvent rattachés à une catégorie que d'éclairer la structure interne des catégories, c'est-à-dire, finalement, de décrire les différents types de relations que peuvent entretenir les sous-catégories constitutives d'une catégorie donnée. Le traitement actuellement proposé de la question de la catégorisation au sens étroit du terme (la question des conditions de rattachement de realia donnés à une catégorie) est donc essentiellement négatif puisque la sémantique cognitive est surtout préoccupée de réfuter le modèle traditionnel dit des conditions nécessaires et suffisantes - modèle de structuration des concepts selon lequel toute mise en oeuvre d'un concept (ou d'une signification) par un sujet requiert que soit satisfait (ou présumé satisfait) un faisceau spécifique de conditions nécessaires et suffisantes - et qu'elle invite à remplacer ce principe unique de structuration par la prise en compte de différents modèles structurants d'origine conceptuelle, baptisés par Lakoff et Johnson modèles cognitifs idéalisés. Or ces modèles autorisent, comme nous le verrons, des types de structuration très variés, du plus contraignant (les "catégories classiques ») au plus lâche (les "catégories radiales»). Ainsi, la structuration logique des concepts et/ou des significations n'est plus guidée ni régulée par un principe universel, mais apparait comme un effet second des modalités diverses selon lesquelles lesdits concepts se sont formés. Comme le dit Lakoff :

La thèse principale de ce livre est que nous organisons notre connaissance à l'aide de structures appelées modèles cognitifs idéalisés, ou MCI, et que les structures catégorielles et les effets prototypiques sont des retombées [by-products] de cette organisation (p. 68) ${ }^{16}$.

On pourrait être tenté d'en conclure avec Kleiber (1990) que, pour définir l'approche de la catégorisation défendue par la sémantique cognitive, il suffit de s'en tenir au plus petit dénominateur commun à ces différents modèles cognitifs, ce qui nous conduirait, en l'occurrence, à retenir le seul principe de la "ressemblance de famille», principe fameux formulé par Wittgenstein et illustré de manière exemplaire par ce que Lakoff et Johnson appellent les «catégories radiales». On pourrait ainsi parler avec Kleiber d'une version 
étendue $d u$ modèle du prototype. Mais ce ne serait pas respecter tout à fait la vision que Lakoff (ou, à un moindre degré, Johnson, puisqu'il se penche moins sur ces problèmes) nous offre de ces questions. D'une part, comme Kleiber lui-même l'indique à plusieurs reprises, le principe de la ressemblance de famille, tel du moins qu'il est exploité par la sémantique cognitive, ne vaut guère que pour la structuration interne des catégories et non pour la catégorisation au sens strict. D'autre part, Lakoff et Johnson ne cherchent nullement à sélectionner un principe unique et ultime de catégorisation que l'on pourrait appeler prototype. Lakoff, en particulier, souligne avec vigueur que le concept de prototype ne définit pas à proprement parler un modèle explicatif. Le prototype n'est jamais pour lui qu'un effet; il ne constitue en aucune façon un principe structurant ${ }^{17}$. Sans écarter tout à fait l'inférence éclairante de Kleiber, nous n'en tiendrons donc pas vraiment compte dans notre présentation des thèses de nos auteurs. Au reste, l'insistance de Lakoff et de Johnson sur la pluralité des modèles cognitifs susceptibles d'expliquer les effets prototypiques peut bien sûr trouver à s'expliquer par l'éclectisme d'un courant de pensée ne dédaignant pas de récupérer au passage l'indéniable efficacité logique des catégories classiques, ou par le souci pédagogique de déployer devant le lecteur la multiplicité des cas étudiés par la linguistique cognitive. Mais, plus fondamentalement, cet attachement à une pluralité de modèles cognitifs nous paraît inséparable du désir de substituer à la problématique traditionnelle de la structuration logique des catégories celle de leur genèse cognitive, dans l'espoir d'extirper toute tentation d'un retour à l'objectivisme ${ }^{18}$.

33 Avec de telles propositions, la sémantique cognitive a indéniablement cassé le jeu de la sémantique traditionnelle. Que l'on se définisse plutôt comme empiriste ou comme rationaliste, que l'on se déclare réaliste, idéaliste ou expérientialiste, et quelle que soit en somme l'interprétation que l'on proposait de ces conditions nécessaires et suffisantes (désormais: CNS), le modèle traditionnel permettait en effet un certain consensus, s'agissant, non pas certes de l'origine de nos connaissances, mais de leur forme et de leur organisation. Il n'en va plus de même avec «l'approche cognitive » qui oblige désormais tout théoricien de la signification à se situer par rapport à une alternative qu'elle a ellemême définie: CNS objectivistes versus modèles cognitifs (ou, pour le dire de façon plus vague et finalement trompeuse, " prototype »)...

On peut toutefois se demander si nos auteurs se sont autant affranchis du modèle des CNS qu'ils veulent bien le croire ou le laisser croire, (cf. infra 3). On observera pour l'heure que la sémantique cognitive dans sa version actuelle (mentaliste) ne développe nulle part une théorie explicite de la catégorisation au sens strict de ce terme, mais seulement un modèle général de la signifiance - entendue comme le produit d'une cognition incarnée complété par un modèle de la structuration interne des catégories. Ainsi, l'essentiel du travail de Lakoff dans son livre consiste à montrer que l'on ne peut se satisfaire du modèle des CNS dans un grand nombre de cas et à mettre en place, à partir d'une étude des cas supposés rebelles, un jeu de concepts "alternatifs", utiles à l'analyse de la structure des catégories.

Ces concepts alternatifs mettent en oeuvre deux types d'objets :

- les « modèles cognitifs idéalisés »;

- les structures catégorielles censées résulter du jeu de ces modèles cognitifs. 


\subsubsection{Les modèles cognitifs idéalisés (désormais : $\mathrm{MCl}$ )}

l'auteur préférant, comme il le reconnaît lui-même (p. 68 et 284) illustrer le concept plutôt que le définir vraiment. "Tout MCI est un tout structuré, une gestalt, utilisant quatre types de principes structurants :

- une structure propositionnelle, comme dans les cadres [frames] de Fillmore,

- une structure de schème d'image, comme dans la grammaire cognitive de Langacker,

- des projections métaphoriques, telles que les décrivent Lakoff et Johnson,

- des projections métonymiques, telles que les décrivent Lakoff et Johnson.

Tout MCI mis en oeuvre structure un espace mental, tel que décrit par Fauconnier» (p. $68)^{19}$

Nous n'évoquerons ici que les modèles propositionnels ${ }^{20}$ qui représentent le seul type de modèle cognitif que nous n'ayons pas encore évoqué et qui, selon Lakoff (p. 113), structurent bon nombre de nos concepts. Ces modèles sont définis négativement, par opposition aux modèles « imaginatifs » :

Par MCI propositionnel, j'entends un modèle qui n'utilise pas de mécanismes [ devices] imaginatifs, c'est-à-dire la métaphore, la métonymie ou l'imagerie mentale (Lakoff : 285)

Plus précisément, il s'agit d'un modèle caractérisant notre connaissance sur un objet conceptuel donné et spécifiant les entités qui le constituent, leurs propriétés ${ }^{21}$ et les relations qui les unissent ${ }^{22}$.

Pour faire mieux comprendre ce qu'il entend par modèles propositionnels, Lakoff nous renvoie, p. 68 et 116, sans beaucoup plus de précision, aux cadres [frames] de Fillmore, ainsi qu'aux cadres de Minsky, aux scripts de Schank et d'Abelson et aux schémas de Rumelhart. Il souligne la compatibilité de ces modèles propositionnels avec le modèle traditionnel des CNS (ainsi qu'avec les modèles computationnels de l'esprit). Il note néanmoins que ces modèles propositionnels peuvent être le théâtre de certains effets prototypiques (cf. infra) qu'il attribue à nos connaissances d'arrière-plan (Searle) et au jeu de valeurs par défaut (Minsky) ou de stéréotypes (Putnam) :

Putnam a utilisé le terme de stéréotype pour désigner à peu près ce que Minsky a décrit comme un cadre avec des valeurs par défaut. Un stéréotype pour Putnam est une représentation mentale idéalisée d'un cas normal, qui peut se révéler inexacte. (...) Dans les stéréotypes de Putnam, les tigres ont des rayures et l'or est jaune, en dépit du fait que l'on rencontre dans le monde réel des tigres sans rayures et de l'or blanc. (p. 116)

Lakoff salue ces apports de Minsky et Putnam. Il reproche cependant à leurs propositions de n'inclure que des modèles propositionnels et de ne pas être capables par conséquent de rendre compte des effets de ce qu'il appelle, avec Johnson, les modèles imaginatifs métaphoriques, métonymiques et à schèmes d'images - (Lakoff : 117).

\subsubsection{Types de structures catégorielles}

De l'action des MCI résultent, comme autant de «retombées » (cf. supra et Lakoff : 68), différents types de structures catégorielles :

- catégories classiques, conformes aux CNS traditionnelles,

- catégories scalaires, 
- modèles métaphoriques et métonymiques,

- catégories radiales.

41 Rappelons qu'une ressemblance de famille suffit à assurer l'appartenance d'un exemplaire à une catégorie, comme il en va dans les « catégories radiales ».

\subsubsection{Catégories classiques}

« La catégorie classique a des frontières nettes [" rigides », p. 153], qui sont définies par des propriétés communes » (Lakoff : 16) ou, en d'autres termes, par des CNS (p. 153).

Ces catégories discrètes, produits de modèles propositionnels, n'en sont pas moins le lieu d'effets prototypiques, certains membres de la catégorie constituant de meilleurs exemplaires que d'autres. Soit, par exemple, le mot célibataire [bachelor] étudié par Fillmore (1982). Il est indéniable que ce mot désigne un homme adulte non marié. Une telle définition, cependant, ne saurait expliquer tout à fait certains usages du mot. Il serait difficile, par exemple, de parler du pape ou de Tarzan comme de célibataires : ce ne sont pas des exemplaires représentatifs de la catégorie. De tels effets prototypiques, souligne Lakoff, ne trouveraient pas d'explication dans une théorie objectiviste non cognitive puisqu'ils résultent de notre aptitude à comparer notre $\mathrm{MCI}$ du concept de célibataire et nos MCI concernant des individus particuliers (le pape, Tarzan). L'application du concept de célibataire est donc tributaire de connaissances d'arrière-plan.

Le terme de mensonge [lie] étudié par Sweetser (1984) offre un autre exemple de catégorie classique donnant lieu à des effets prototypiques en vertu d'un « MCI de l'interaction sociale et linguistique » qui, dans certains cas, dispense les locuteurs de dire la vérité (Lakoff : 72). Nous savons tous que le jeu des conventions sociales permet de ne pas considérer certains « mensonges factuels » (mensonges de convenance, pieux mensonges, blagues, plaisanteries, exagérations, récits de fiction, erreurs pures et simples...) comme de véritables mensonges. Il n'en reste pas moins, estime Lakoff, que le mensonge peut et doit être défini en termes de CNS, comme l'a montré Sweetser en réponse à Coleman et Kay.

\subsubsection{Catégories scalaires}

Relèvent de ce deuxième type des « catégories » telles que homme grand [tall man] ou rouge qui « présentent de manière inhérente des degrés d'appartenance, des frontières floues et des membres centraux dont le degré d'appartenance catégorielle (sur une échelle de zéro à un) est de un. » (Lakoff : 56). On rejoint ici les travaux de Lotfi Zadeh sur les ensembles flous : «Dans un ensemble classique, chaque chose est soit à l'intérieur de l'ensemble (sa valeur d'appartenance est de 1), soit à l'extérieur de cet ensemble (sa valeur d'appartenance est de 0). Dans un ensemble flou (...), des valeurs supplémentaires sont autorisées entre 0 et $1 »$ (Lakoff : 21-22).

De même, « certains hommes ne sont ni franchement grands ni franchement petits, mais plutôt de taille moyenne - ils sont grands à un certain degré. » (Lakoff : 22). On pourrait faire les mêmes remarques à propos des termes de couleur.

Les catégories scalaires débouchent donc constamment sur des effets prototypiques. 


\subsubsection{Modèles métaphoriques et métonymiques ${ }^{23}$}

\subsubsection{Catégories radiales}

"Une structure radiale est une structure où il existe un cas central et des variations conventionnalisées de ce cas central qui ne peuvent pas être prédites par des règles générales (Lakoff : 84) En d'autres termes, dans un modèle radial, les extensions de la sous-catégorie centrale "ne sont pas engendrées à partir du modèle central par des règles générales, mais sont étendues par convention et doivent être apprises une par une. Mais les extensions ne sont en aucune façon le fait du hasard» (Lakoff : 91) ${ }^{24}$. Lakoff cite pour exemples de ces structures la "catégorie conceptuelle" mother ( $c f$. infra pour une contre-analyse) et les classificateurs du dyirbal qui lui ont inspiré le titre de son ouvrage.

\section{Les limites de l'expérientialisme individualiste de Lakoff et Johnson}

cognitive auront en commun de remettre en question son mentalisme, d'en montrer les conséquences néfastes pour l'analyse linguistique et de jeter les bases d'une approche « alternative » de type médiationniste.

Nous nous attacherons en premier lieu aux «structures symboliques » censées fonder, chez Lakoff et Johnson, le monde des significations humaines, avant de nous concentrer, dans la partie suivante, sur les questions de la catégorisation et de la structuration catégorielle.

\subsection{Les « structures directement signifiantes » : niveau de base et schèmes d'images}

Commençons par préciser que nous partageons la thèse première de Lakoff et Johnson selon laquelle la théorie de la signification et, plus généralement, la théorie du langage, doit en toute chose s'attacher à spécifier les modalités selon lesquelles les formes linguistiques acquièrent leur signification pour les sujets humains. Nous accorderons volontiers également que toute activité symbolique humaine dérive en dernière analyse d'expériences d'ordre sensori-moteur qui la rendent signifiante pour les sujets.

51 Nous ne souscrirons pas en revanche à la thèse selon laquelle la structure de l'expérience préconceptuelle sensori-motrice, associée avec des mécanismes « imaginatifs ", suffirait à rendre compte de la totalité des phénomènes sémantiques, en sorte que tout phénomène d'ordre symbolique ne pourrait que reconduire inlassablement la structure de cette expérience chronologiquement première. L'expérience sensori-motrice est d'une certaine manière à l'origine du symbolisme, mais l'expérience sensori-motrice n'est pas le symbolisme. En d'autres termes : au commencement était le corps, certes, mais, s'agissant de l'homme au moins, considéré dans l'histoire individuelle comme dans l'histoire des groupes, le verbe (et, plus généralement, le signe) eut tôt fait de bouleverser la donne.

\subsubsection{Le niveau de base}

On s'étonnera d'abord de l'insistance des cognitivistes classiques à vouloir définir un niveau privilégié et invariant d'interaction entre l'homme et son environnement. L'idée 
même de niveau implique normalement en effet un ordre de phénomènes bien circonscrit et plus ou moins constant d'un sujet à l'autre, d'un groupe à l'autre, d'une culture à l'autre. On est alors en droit d'objecter que le niveau d'interaction entre les sujets cognitifs et leur environnement devrait au moins fluctuer selon l'expérience des sujets, leur âge, leur niveau d'expertise et, plus encore, les activités dans lesquelles ils se trouvent engagés; et qu'en conséquence il n'existe pas à proprement parler un niveau privilégié et constant d'interaction de l'homme avec son environnement. Ainsi, dans l'ordre de l'expérience humaine, la signification «mammifère » - nous parlerons pour notre part de signification car on peut douter de l'existence d'une "catégorie prélinguistique » correspondante -, ou, à l'inverse celle de «normande» ou de " mérinos ", peut se révéler tout aussi pertinente que celle de " vache » ou de « mouton », chaque type de signification pouvant être amené à jouer un rôle privilégié selon le type d'activité dans lequel le sujet se trouve engagé, autrement dit selon la forme d'interaction qu'il entretient avec son environnement. Négliger ces variations nous conduirait à faire prévaloir un type de comportement contre un autre, voire un type d'humanité contre un autre. Lakoff (p. 37) cite d'ailleurs des réflexions de Berlin lui-même s'efforçant de prendre en compte une certaine variabilité du niveau d'interaction, mais ces concessions ne nous convainquent nullement de la nécessité de conserver la notion de niveau de base entendue comme une sorte d'invariant cognitif interindividuel, inter-âges et intercontextes, fût-ce en imaginant avec Berlin des espèces de " clauses suspensives ».

On observera surtout que le niveau de base constitue un concept superflu dès lors que l'on n'adhère plus aux postulats mentalistes de la sémantique cognitive et que l'on n'a plus besoin d'ancrer la cognition dans une expérience exclusivement et définitivement préconceptuelle et prélinguistique.

Nous nous accordons volontiers avec Lakoff et Johnson sur une certaine précellence du sensori-moteur dans la conceptualisation humaine, à condition d'entendre cette précellence comme une antériorité chronologique des expériences sensori-motrices sur les expériences sémiotiques (i.e. impliquant une forme quelconque de langage), tant dans l'histoire de l'individu que dans celle des groupes humains. Nous rejetons en revanche l'existence d'un «niveau » privilégié - défini du reste de manière plutôt circulaire auquel tous les autres niveaux seraient nécessairement directement et constamment reliés et nous contesterons de même le postulat, cher à nos auteurs, selon lequel seule l'expérience sensori-motrice serait apte à donner du sens aux expériences humaines. Le concept de niveau de base, en réduisant l'homme aux dimensions d'un être sensorimoteur qui ne connaîtrait le monde que par son corps, nous semble reconduire le mythe d'une humanité adamique libérée du temps, de l'histoire et des signes. Or l'homme est certainement un animal beaucoup plus complexe et subtil que ne veut le croire la sémantique cognitive.

\subsubsection{Les schèmes d'images}

La thèse de l'existence de schèmes préconceptuels n'est pas par elle-même très originale pour qui s'intéresse aux sciences cognitives ou aux neurosciences et l'expression de schèmes d'images pourrait, à ce titre, être considérée comme une autre dénomination pour des phénomènes d'activation neuronale dont l'existence est aujourd'hui bien connue. L'originalité de Johnson sur ce point réside plutôt dans le caractère phénoménologique des descriptions qu'il propose de ses propres schèmes d'images et, plus encore, dans la place considérable qu'il assigne à leurs projections métaphoriques et métonymiques dans 
son analyse de la conceptualisation humaine. Loin de nous donc l'idée de contester l'existence de schèmes préconceptuels liés à nos activités sensori-motrices ou de nier que ces schèmes jouent un rôle crucial pour la cognition humaine comme pour les autres espèces animales. Il reste que la mise en évidence de schèmes préconceptuels ne nous dispense pas d'une généalogie proprement sémiotique des significations.

Ainsi, nous conviendrons volontiers que l'interprétation d'un énoncé implique toujours, à un certain degré, la mise en jeu d'expériences préconceptuelles et que le concept de force ne saurait prendre sens pour un sujet sans une expérience préconceptuelle de la force (Johnson: 13). L'influence de l'objectivisme aux Etats-Unis a été si pesante qu'il peut être utile de rappeler certaines vérités de bon sens. Il existe toutefois un abîme entre une expérience préconceptuelle quelconque et le concept qu'on pourrait être tenté de lui rattacher et il importe donc de souligner, contre Johnson, qu'une expérience préconceptuelle comme celle de la force ne saurait suffire à déterminer le concept correspondant, à moins d'être prêt à postuler que tout organisme faisant l'expérience de ce que nous appelons la force dispose ipso facto d'un concept de force...

La sous-estimation de l'écart entre expérience préconceptuelle et concept se révèle particulièrement lourde de conséquences lorsque Johnson évoque les projections métaphoriques de ces schèmes, comme nous le verrons plus loin. Contentons-nous d'observer pour l'instant que l'analyse du schème d'image de la force qui nous est proposée $\mathrm{p} .43 \mathrm{sq}$ fait appel à une pensée conceptuelle passablement complexe et élaborée et qu'il serait fort hasardeux de prétendre que les sujets cognitifs disposent d'un schème préconceptuel de la force présentant les traits que Johnson lui prête :

Premièrement, la force est toujours éprouvée dans l'interaction (...) Deuxièmement, notre expérience de la force implique ordinairement le mouvement à travers l'espace d'un objet (d'une masse) dans une certaine direction (...) Troisièmement, une force suit typiquement une trajectoire unique [there is typically a single path of motion] (...) Quatrièmement, les forces ont des origines ou sources et, parce qu'elles sont directionnelles, des agents peuvent les diriger vers des cibles (...) Cinquièmement, les forces ont des degrés de puissance ou d'intensité (...) Sixièmement, $\mathrm{du}$ fait que nous éprouvons la force à travers l'interaction, elle met toujours en jeu une structure ou une séquence de causalité (... $)^{25}$

On peut indéniablement attribuer de telles caractéristiques aux forces qui s'exercent dans la nature. Il nous paraît totalement exclu en revanche que les sujets cognitifs soient en mesure de les isoler en tant que telles à un stade préconceptuel. Avant toute acquisition du langage, en effet, « les forces qu'ils éprouvent » se présentent aux sujets comme autant de « touts » synthétiques inanalysables. Nous estimons par conséquent que les propriétés qui ont retenu l'attention de Johnson ne peuvent être dégagées que rétrospectivement, à la lumière du langage et de la forme spécifique d'analyse et de culture qu'il rend possible.

Nous ajouterons que nos schèmes préconceptuels ne peuvent qu'être sensiblement changés par le langage. Il est vrai que le rôle des systèmes linguistiques dans la constitution des schèmes d'images n'est pas totalement nié par Johnson: «Ces patterns existent pour nous prélinguistiquement, bien qu'ils puissent être notablement affinés et élaborés par l'effet de l'acquisition du langage et du système conceptuel auquel le langage donne accès » (p. 48). On remarquera cependant que le rôle attribué au langage reste limité et marginal («affinés et élaborés »). Pour nous, au contraire, non seulement nos schèmes préconceptuels et, d'une manière générale, notre perception elle-même, sont en profondeur reconfigurés par le langage, mais il existe une véritable solution de continuité ( $c f$. infra) entre les schèmes que nous développons individuellement de manière pré- ou 
extralinguistique et les concepts et expressions linguistiques auxquels nous accédons grâce à notre initiation au langage et à la culture d'un environnement humain spécifique.

\subsection{Les projections métaphoriques et métonymiques} sensori-motrice, de même nous souscrivons depuis longtemps à la thèse selon laquelle le développement de la pensée dans notre espèce doit beaucoup à l'existence de modèles ou de matrices que l'on peut qualifier, si on le souhaite, de «métaphoriques » (et aussi, à un degré qui reste à déterminer, de modèles ou de matrices "métonymiques »), lesquels trouvent d'une certaine manière leur origine dans les expériences de la vie humaine. Nous considérons même cette thèse, qui est au cœur du célèbre livre de Lakoff et Johnson (1980), mais aussi de travaux antérieurs de Guiraud sur les protosémantismes (cf. notamment 1967), comme l'un des acquis fondamentaux de la réflexion de ces auteurs, même si nous croyons devoir la réinterpréter d'une manière sensiblement différente ( $c f$. Nyckees 2000c, 2000d, à paraitre b), et 1998a (chap. 7)).

Notre désaccord avec Lakoff et Johnson n'en est pas moins profond. Il porte d'abord, comme on peut s'y attendre, sur l'occultation du rôle du langage et de la culture dans leur analyse des projections métaphoriques et métonymiques. Mais il concerne également la thèse de Johnson selon laquelle il serait possible de fonder des raisonnements élaborés et valides sur la projection de ses "schémes d'images » d'un domaine d'expérience à un autre.

\subsubsection{Pas de métaphores ni de métonymies sans langage}

62 Nous commencerons donc par réfuter l'idée, chère à Johnson, selon laquelle des "projections ", notamment métaphoriques, pourraient s'effectuer sans langage et que la plupart d'entre elles s'effectueraient réellement à un niveau préconceptuel et prélinguistique ${ }^{26}$. Il n'existe pas en effet de métaphore ou de métonymie ni, de manière générale, de trope ou de sens figuré sans langage, ne serait-ce que parce que toute figure et tout trope présupposent par définition une expression linguistique. Il n'y a donc pas lieu, selon nous, de parler de métaphore et de métonymie pour désigner des phénomènes purement conceptuels ou préconceptuels comme le font Lakoff et Johnson ${ }^{27}$. Tout au plus peut-on parler en pareil cas d'heuristiques mettant en œuvre des intuitions, des jugements et même des raisonnements élémentaires fondés sur l'analogie (assimilations) ou sur la contiguïté (généralisations, extrapolations).

Ainsi pourra-t-on citer au titre des analogies préconceptuelles (non métaphoriques) ce que nous appellerons des "conduites assimilatrices préconceptuelles»- extensions analogiques (préconceptuelles) de schèmes sensori-moteurs -, comme celles que met en œuvre un enfant lorsqu'il utilise dans un nouveau contexte, face à de nouveaux objets, et en l'adaptant à ces nouvelles conditions, un savoir-faire sensori-moteur acquis dans d'autres circonstances.

Nous reconnaitrons volontiers également l'existence d'une forme de "symbolisme" analogique d'ordre émotionnel préexistant au langage verbal (même si la pratique de la conversation, de l'écriture ou de la lecture peut en renforcer les effets), mais nous nous refuserons à parler en pareil cas de métaphore. Nul doute, par exemple, que, de façon assez "naturelle», une photographie lumineuse dans une séquence filmique éveille 
plutôt des anticipations optimistes (sauf ironie ou raffinement de cruauté comme au début de Shining), alors qu'une photographie sombre encouragera plutôt des anticipations pessimistes. Il ne s'agit nullement néanmoins de métaphores dans ces deux cas. De même, la vue du masque Kifwebe évoqué par Johnson, p. 80 sq, avec sa composition particulière de formes, de lignes et de couleurs, pourrait susciter en nous une certaine impression. Admettons, pour les besoins de la démonstration, que cette composition nous affecterait de la même manière si nous ne disposions pas du langage et imaginons qu'un observateur ait en la circonstance accès à nos états mentaux. Supposons enfin que cet observateur trouve bon de traduire l'impression ressentie par le mot équilibre [balance ${ }^{28}$. Serait-il pour autant fondé à affirmer que nous avons nous-même procédé inconsciemment à une projection métaphorique de manière préconceptuell ${ }^{29}$, comme le soutient Johnson?

On devra donc distinguer soigneusement deux types de cas lorsque nos auteurs parlent de métaphore ou de métonymie: les cas où l'expression est utilisée indûment pour renvoyer à un processus fondé sur l'analogie ou la contiguïté et censé se dérouler indépendamment du langage, et ceux où l'expression est utilisée à bon droit pour renvoyer à des processus d'ordre métaphorique ou métonymique mettant en œuvre le langage. Malheureusement, de nombreux usages de ces termes par Lakoff et Johnson correspondent au premier de ces types. C'est ainsi, par exemple, qu'aucun des prétendus "modèles métonymiques" énumérés par Lakoff, p. 90 (cf., supra, le paragraphe qui leur est consacré) ne nous parait mériter le qualificatif de métonymique. Il s'agit en effet exclusivement de mécanismes de raisonnement par extrapolation ou généralisation et, plus précisément, d'heuristiques fondées sur la présomption que ce qui est vrai d'une sous-catégorie ou d'un échantillon d'une catégorie - surtout s'il s'agit d'une sous-catégorie ou d'un exemplaire tenu pour particulièrement représentatif - pourrait l'être également d'une autre partie ou d'un autre exemplaire de cette même catégorie, voire de la catégorie tout entière.

On acceptera en revanche, de parler avec Lakoff de métonymie lorsque, par exemple, une institution est désignée par le nom du lieu où elle est située. Ce type de métonymie est d'ailleurs lui-même reconductible à un principe général, comme le note Lakoff. Or, comme il le reconnaît également (p. 78), de tels principes généraux peuvent être propres à une langue ${ }^{30}$. Il reste que, dans ce dernier cas, la relation de « contiguïté » entre les deux significations relève clairement, non pas du discours, mais de la langue elle-même ou, plus exactement, de la compétence linguistique des locuteurs concernés - pour plus de précisions sur ces questions, $c$. à paraitre b) ${ }^{31}$. Ajoutons qu'il convient de continuer à parler de deux significations distinctes pour la forme linguistique considérée (par ex., pour la Maison Blanche, le lieu et l'institution), même si ces deux significations entretiennent une relation privilégiée ( $c f$. ., infra, « la question de la polysémie »).

Délaissant les problèmes spécifiques soulevés par les métonymies, nous nous concentrerons à présent sur ceux que posent les projections métaphoriques, longuement étudiées par Johnson.

\subsubsection{Des métaphores « naturelles » très culturelles}

68 Notre deuxième point de désaccord avec le traitement des métaphores proposé par la sémantique cognitive actuelle (rappelons qu'il s'agit toujours de métaphores fortement conventionnelles, inscrites en langue) porte sur leur caractère prétendûment naturel. Il nous semble en effet que la sémantique cognitive occulte ou minimise systématiquement la dimension culturelle et historique des processus sémantiques, et en particulier 
métaphoriques, en prétendant rabattre tout phénomène sémantique sur une expérience corporelle immédiatement accessible (d'où le caractère paradoxal des longues explications proposées pour rendre compte de la motivation des métaphores ${ }^{32}$ ) et en voulant rendre compte dans les mêmes termes: (i) du sentiment linguistique des locuteurs concernant le sens figuré lexical synchronique (i.e. une relation synchronique de type analogique entre deux significations d'un même mot) et (ii) des processus historiques susceptibles d'expliquer ce sens figuré lexical.

Il s'agit en effet, pour Lakoff comme pour Johnson, de «montrer que la métaphore est naturelle en ce sens qu'elle est motivée par la structure de notre expérience » (Lakoff : 276). Or l'expérience invoquée par nos auteurs est exclusivement de nature corporelle: «Les métaphores ne sont pas arbitraires, mais sont elles-mêmes motivées par des structures inhérentes à notre expérience corporelle quotidienne » (Lakoff : 275). C'est généralement une certaine corrélation structurelle dans notre expérience sensori-motrice qui se trouve invoquée pour motiver la métaphore, dans son ensemble comme dans ses détails (Sweetser allègue toutefois, dans certains cas, une analogie "irréductible », cf. infra). Nous soutiendrons au contraire que, d'une manière générale, la motivation expérientielle des métaphores représente moins un phénomène transparent et immédiatement perceptible pour les locuteurs qu'un héritage conceptuel issu de notre histoire linguistique et culturelle, et que ce n'est guère qu'à ce titre que nombre de métaphores peuvent constituer des réalités familières. Ainsi, les exemples donnés par Lakoff pour illustrer la «métaphore » LE PLUS EST EN HAUT ${ }^{33}$, ou, en tout cas, la plupart d'entre eux, seraient mieux expliqués, nous semble-t-il, par des techniques de représentation graphique :

Le taux de criminalité continue à monter. Le nombre de livres publiés chaque année continue de s'élever. Ce titre a de nouveau chuté. Nos ventes ont baissé l'an dernier. Vous obtiendrez un intérêt plus élevé avec eux. Nos réserves financières ne peuvent pas être plus basses. (Lakoff : 276)

Il est vrai que les techniques de représentation graphique s'expliquent sans doute ellesmêmes en dernière analyse par la corrélation invoquée par Lakoff. Elles nous semblent présupposer en effet l'existence d'instruments de mesure gradués tels que, à date ancienne, piquets, toises, jauges, récipients..., manifestement en rapport avec des pratiques et des techniques élémentaires d'accumulation et de stockage qui vérifient la fameuse corrélation (constitution de tas, de réserves, de greniers, etc.). Mais cette corrélation n'a pu connaître, précisément, une telle efficacité que parce qu'elle s'est trouvée relayée, et renouvelée, par des dispositifs techniques. Si, pour nous aujourd'hui, "LE PLUS EST EN HAUT », c'est bien parce que les développements des techniques de mesure ont permis cette équation, qu'ils l'ont entretenue, qu'ils ne l'ont pas en somme périmée ou occultée, à la différence de ce qu'il a pu advenir d'autres métaphores à travers l'histoire linguistique. Les explications de Lakoff et de Johnson ne représentent donc qu'un raccourci. Elles doivent être sérieusement affinées et prendre en compte bien d'autres étapes de l'évolution. Ainsi, si la "métaphore» LE PLUS EST EN HAUT paraît naturelle, c'est d'abord du fait de la culture, même si - nous en conviendrons sans difficulté - elle s'enracine in fine dans les propriétés d'un certain type d'interaction sensori-motrice entre l'homme et son environnement.

71 Cet exemple devrait nous inciter à la prudence dans l'interprétation historique de certains faits synchroniques et, en particulier, de certaines polysémies : ce n'est pas parce que, en synchronie, nous percevons à bon droit comme métaphoriques certaines relations entre significations que lesdites relations ont nécessairement procédé historiquement de 
projections métaphoriques véritables, impliquant de la part de leurs promoteurs une sorte de saut conceptuel ${ }^{34}$. Non seulement bien des causes autres que métaphoriques peuvent être envisageables pour expliquer tel changement de sens à l'origine d'une polysémie donnée, mais les polysémies résultent sans doute bien souvent d'évolutions continues, sans rupture sensible, et dont les promoteurs mêmes n'ont pas eu une claire conscience ( $c$. Nyckees 1997a; 1998a: 139 sq; et surtout 2006; mais aussi 4. infra). La sémantique cognitive n'a que trop tendance à ignorer ces cas parce qu'elle veut toujours considérer le sentiment linguistique des locuteurs comme une bonne entrée pour éclairer les changements de sens passés et qu'elle n'imagine pas qu'une partie de la machinerie puisse leur être dérobée. Attachée à des explications exclusivement conceptuelles des phénomènes sémantiques reposant sur des processus mentaux invariants dans le temps et l'espace, elle ne peut manquer d'occulter la dimension historique des significations linguistiques.

72 Pour en revenir au thème, cher à Johnson comme à Sweetser, de la naturalité des métaphores, le (prétendu) schème d'image préconceptuel de la BALANCE ou de l'ÉQUILIBRE [anglais BALANCE] (Johnson: $121 \mathrm{sq}$ ) et celui des LIENS [LINKS] (ibid.: $117 \mathrm{sq}$ ) nous offriraient d'autres illustrations de l'importance des artefacts dans la cognition humaine et le langage humain. Comme le note Lakoff (p. 274), «l'esclavage est compris comme liens, chaînes [bondage] et la liberté comme l'absence d'entrave.» Ne faut-il voir dans ce symbolisme que le produit d'une vague analogie perçue par notre esprit, comme le voudraient nos auteurs? Ne faut-il pas plutôt y reconnaître l'effet de la sédimentation dans nos langues d'une expérience éminemment concrète et socio-culturellement déterminante, celle d'artefacts - chaînes et cordes - parfaitement intégrés à nos sociétés humaines depuis la nuit des temps ${ }^{35}$ ? Telle n'est pas malheureusement l'orientation choisie par Johnson qui ne cesse de "naturaliser" ses "concepts métaphoriques", comme s'ils n'avaient pas d'histoire ( $c$. par exemple ses développements sur la notion d'équilibre systémique ou sur celle d'équilibre psychologique, p. 87-88).

Le schème du CHEMIN [PATH] (ibid. : 113 sq) illustrerait également le poids, dans notre cognition et notre langage, des artefacts ou des naturalia aménagés ou instrumentalisés par l'homme. Il n'en irait pas autrement du schème des CYCLES (ibid. : 119 sq), dont le rapport avec les roues (et peut-être les meules) ne fait guère de doute; du « schème » du CONTENANT [CONTAINER] (cf. liste p. 126), manifestement en relation avec l'usage immémorial de la poterie; ou encore du « schème » CENTRE / PÉRIPHÉRIE (ibid. : 124 sq) en rapport probable avec divers artefacts et pratiques socio-techniques attestés à date très ancienne : outre la roue avec sa jante et son moyeu, on citera ainsi les foyers dont la chaleur se propage en décroissant à mesure que l'on s'éloigne du centre, les clôtures et les palissades délimitant les frontières d'un lieu, ou le confinement des déchets et la relégation de certains animaux à la périphérie des zones d'habitation.

Tous ces exemples plaident selon nous en faveur d'une approche située et externalisée de la cognition (cf. notre introduction) où le langage joue un rôle de médiateur et de catalyseur. Ce ne sont pas en effet des expériences purement préconceptuelles d'ordre cénesthésique ou kinesthésique qui, du seul fait de leur existence, ont pu suffire à structurer notre compréhension d'un certain nombre de domaines que nous percevrions grâce à elles en termes de relations contenant/contenu ou en termes d'équilibre. Ce sont bien plutôt, à l'inverse, des signes linguistiques désignant des artefacts (récipients, balances, par exemple) ou les expériences techniques correspondantes (fabrication et utilisation de poteries ou de balances) qui nous ont permis d'analyser nos expériences préconceptuelles 
et de renouveler la compréhension que nous en avions. C'est en effet la technique qui nous sert à penser nos expériences corporelles et mentales (de même que, d'une manière générale, c'est l'autre et le lointain qui nous permettent de penser le moi, le nous, le proche et le semblable), bien plutôt que l'inverse. Ainsi, depuis quatre siècles au moins et, sans doute depuis fort longtemps, les techniques jugées à chaque époque les plus sophistiquées et les plus admirables ont permis aux hommes de penser et de modéliser l'esprit humain, ses propriétés et ses activités: mécanismes d'horlogerie, automates, techniques de télécommunication, ordinateurs...

Lakoff et Johnson, dans leur ouvrage de 1980, ont brillamment illustré la thèse selon laquelle ce qui présente pour nous une structure bien définie nous aide à appréhender (par la métaphore en particulier) ce qui ne présente pas de structure manifeste (cf. encore Lakoff : 303). On peut regretter dans ces conditions qu'ils n'aient pas mieux perçu le rôle structurant des techniques pour la cognition humaine. L'outil, en effet, parce que sa fabrication requiert des composants clairement articulés et décomposables et parce que ses usages impliquent des séquences précisément définies, propices à l'apprentissage, représente assurément l'un de nos meilleurs pourvoyeurs en modèles métaphoriques.

\subsubsection{La projection des schèmes d'images}

76 Tout l'argumentaire de Johnson (1987) en faveur d'une cognition mentaliste repose sur sa démonstration de la thèse selon laquelle il serait possible de projeter des schèmes d'images d'un domaine d'expérience sur un autre et d'en tirer des inférences valides pour le domaine cible. Or il nous semble que l'ensemble de cette démonstration est affecté par un vice logique qui en compromet irrémédiablement les conclusions. Nous montrerons ainsi que Johnson ne peut croire que les SI, à eux seuls, rendent possibles des inférences de notre système conceptuel (et donc nos opérations de raisonnement) que parce qu'il a préalablement infiltré, sans même s'en rendre compte, dans l'expérience présumée prélinguistique et préconceptuelle qu'il décrit, les catégories conceptuelles d'une expérience informée par le langage. S'il en allait autrement d'ailleurs, n'importe quel animal serait susceptible de conduire des raisonnements sophistiqués du type de ceux que les humains dotés de langage mettent en œuvre dans leur discours et leur pensée.

77 Johnson travaille en effet avec un concept non critique et ambigu d'expérience qui le conduit à confondre états mentaux et realia ${ }^{36}$. Il ne distingue jamais dans la description des schèmes d'images entre ce qu'on peut appeler l'objet vécu, c'est-à-dire l'objet tel que ressaisi et " vécu » par le sujet, « objet » situé manifestement du côté des états mentaux, et ce que nous appellerons l'objet expériencé c'est-à-dire l'objet, ou plutôt l'aspect du monde ${ }^{37}$, tel que livré à l'expérience du sujet et clairement situé du côté du réel.

78 Cette indistinction, entre objet vécu et objet expériencé ne portait pas à conséquence lorsque Johnson ne se proposait que d'illustrer le concept de schème d'image en analysant certains exemples de son choix et qu'il adoptait le point de vue d'un observateur extérieur mettant en relation des structures mentales supposées du sujet avec certains états de choses. Une telle assimilation devient en revanche hautement problématique lorsque, à partir, notamment, du chapitre 3, Johnson entreprend de mettre en évidence le rôle des projections métaphoriques dans le raisonnement et la compréhension humaine et que, réutilisant «tout naturellement » les exemples traités antérieurement pour illustrer le concept de SI, il entend démontrer le profit que notre raisonnement peut tirer du transfert des caractéristiques observées d'un domaine 
d'expérience à un autre. Il oublie en effet tout simplement que les descriptions de schèmes d'images qu'il nous avait jusqu'alors proposées (schèmes de la FORCE, du CONTENANT, du CHEMIN, etc.) ne prenaient pas pour objet en réalité les états mentaux du sujet, mais seulement les realia donnant lieu à ces états mentaux - à moins, bien sûr, que, par un retour inattendu au réalisme le plus pur, il ne soit prêt à soutenir que l'état mental suscité par une perception a la même structure, la même forme, que l'aspect du monde correspondant. Dès lors, la position en surplomb de Johnson, tout à la fois à l'intérieur et à l'extérieur du sujet cognitif, tel un romancier omniscient, cesse d'être tenable, l'objectif n'étant plus de cerner ce qui dans le monde a pu déclencher la formation de tel schème d'image, mais d'observer le processus de projection métaphorique lui-même, ce qui, en bonne règle, devrait impliquer de s'en tenir exclusivement au point de vue du sujet cognitif. Or, à l'intérieur d'un système cognitif, ce ne sont évidemment pas les caractéristiques des realia expériencés que l'on doit rencontrer, mais bien des états mentaux dotés d'une tout autre structure.

Par là même, c'est tout un peuple de catégories sophistiquées, hautement sémiotisées et informées par le langage, qui se trouve réinjecté dans une couche d'expérience présentée par Johnson comme prélinguistique et préconceptuelle ${ }^{38}$. On ne sera pas surpris, à ce compte, de voir le schème préconceptuel du CONTENANT crédité du pouvoir de fonder nos raisonnements et jusqu'aux lois de la logique par un simple transfert métaphorique ( cf. supra).

80 Une telle confusion n'aurait pas été possible sans une méprise plus profonde sur la nature de nos «connaissances" préconceptuelles. Il nous semble en effet qu'en tant que structures préconceptuelles, nos schèmes sensori-moteurs ne sauraient correspondre par définition qu'à des savoir-faire (know-how), c'est-à-dire à des connaissances ou des aptitudes "contextuellement dépendantes", non mobilisables - à l'état de veille du moins - en l'absence de contextes déclencheurs appropriés ${ }^{39}$. Il s'ensuit que nos schèmes ne devraient pas pouvoir se prêter à des transferts d'un domaine d'expérience à un autre, puisque ces opérations requerraient leur autonomie effective à l'égard des contextes déclencheurs. Or, en prétendant qu'ils peuvent faire l'objet de projections métaphoriques, Johnson traite ipso facto ces schèmes préconceptuels comme des savoirs (know-what $)^{40}$, i.e. comme des connaissances de type conceptuel, autonomisables comme telles et mobilisables à volonté.

81 C'est un vice de raisonnement comparable que l'on retrouve lorsque Johnson, afin de montrer la prégnance du schème d'orientation INTÉRIEUR/EXTÉRIEUR [IN/ OUT] ( $p$. 30-31), invite le lecteur à s'imaginer dans les premiers instants qui suivent le réveil et qu'il attire son attention sur les expressions couramment utilisées pour dépeindre cet état bien particulier : « Vous sortez peu à peu de votre état d'engourdissement » note-t-il par exemple ["You gradually emerge out of your stupor »]. Or, on observera que, pour qu'un sujet puisse se doter de la représentation correspondant à une telle phrase, il lui faudrait déjà avoir mené à son terme la conceptualisation d'un état intérieur. Un « humain » qui, par hypothèse, serait dépourvu de tout langage et ne serait doté que d'un schème d'orientation IN/OUT, le rendant apte à discriminer un intérieur et un extérieur, ainsi que de capacités d'assimilation analogique ( $c f$. supra), ne saurait selon nous y parvenir puisque :

1. il serait incapable de déconnecter l'opposition intérieur/extérieur des contextes déclencheurs d'ordre sensori-moteur qui la rendent pertinente pour lui ; 
2. il ne pourrait isoler la stupor comme un état spécifique et défini, se prêtant à certaines projections métaphoriques.

82 Ces difficultés nous paraissent signer l'échec de la sémantique cognitive à rendre compte de la conceptualisation humaine. On serait en droit d'attendre en effet du principe de projection métaphorique des schèmes d'images un éclairage sur la constitution de concepts abstraits à partir de concepts ou de schèmes concrets (sensori-moteurs). Lakoff et Johnson laissent d'ailleurs volontiers entendre que la sémantique cognitive marque un progrès sensible dans la compréhension de la genèse de la conceptualisation humaine. Or, à y regarder de près, les exemples traités présupposent toujours que le domaine abstrait a d'emblée été donné aux sujets - sans avoir à être construit - comme le suggère d'ailleurs le terme même de projection, ce qui restreint considérablement la portée et l'intérêt de leurs analyses. Ainsi, la «métaphore » LE PLUS EST EN HAUT présuppose que la notion de quantité a déjà été élaborée. De manière analogue, la phrase You gradually emerge out of your stupor présuppose la conceptualisation préalable de la «stupor». La sémantique cognitive ne nous apprend malheureusement rien dans le premier cas sur la manière dont la notion de quantité (LE PLUS) s'est trouvée construite dans notre espèce, ni, dans le second, sur les raisons pour lesquelles un état tel que la stupor peut être interprété comme un contenant par des sujets. Il apparaît donc que cette aproche n'est guère capable de rendre compte de l'émergence de sens figurés abstraits. Il est vrai qu'une généalogie authentique de nos « concepts » requiert sans nul doute une investigation diachronique, une archéologie des significations linguistiques. C'est du moins notre opinion ${ }^{41}$. Une sémantique (ou une psychologie) ne voulant prendre en considération que les capacités cognitives susceptibles de se développer au cours d'une seule vie humaine ne saurait y parvenir. Or la théorie de la signification défendue par la sémantique cognitive (cf.notre première partie) oblige à considérer que les sujets cognitifs possèdent dans leur expérience corporelle (sensori-motrice) tous les éléments requis pour comprendre et élaborer les métaphores (et les concepts) dont ils font usage. Elle ne prévoit pas que l'origine des phénomènes sémantiques observables puisse ne pas être immédiatement accessible à leur conscience ou à leur intuition.

83 La porte est donc d'emblée fermée à de véritables élucidations historiques des grandes métaphores structurant notre compréhension. C'est ainsi que la sémantique cognitive renonce finalement à expliquer les métaphores, allant même parfois, pour justifier cet abandon, jusqu'à invoquer, avec Sweetser (1990), l'irréductibilité de certains processus métaphoriques ou, avec Johnson, la créativité de la métaphore. Nos auteurs croient d'ailleurs trouver là un argument supplémentaire en faveur de leur thèse selon laquelle la compréhension du langage naturel requiert la compréhension humaine, sans prendre garde au fait qu'un tel argument ruine leurs ambitions explicatives.

Ainsi, Sweetser conteste la possiblité de dégager une corrélation expérientielle pour toutes les métaphores. Aucune corrélation, selon elle, ne saurait rendre compte de l'emploi abstrait de bitter dans bitter anger ou de sweet dans sweet personality. La seule explication retenue est une analogie : la colère est déplaisante pour notre vie émotionnelle tout comme une saveur amère pour nos papilles gustatives (p. 29). Ces métaphores sont certes " motivées » selon elle par une métaphore générale qui permet de concevoir le mental dans les termes de nos expériences corporelles (la Mind as Body Metaphor, isolée par Kurath), métaphore motivée elle-même dans son ensemble par des corrélations partielles. Mais, de l'avis de Sweetser, la Mind-as-Body Metaphor ne saurait expliquer à elle seule l'application sweet $1 \rightarrow$ sweet 2 ou bitter $1 \rightarrow$ bitter 2 (p. 30). 

capable de créer une similitude qui n'existe pas préalablement dans une expérience indépendante du sujet cognitif. Le problème est que cette similitude ne semble pas davantage exister dans l'expérience entendue en un sens riche, "expérientialiste» précisément, comme co-détermination et co-spécification du sujet et de l'objet, et qu'elle paraît plutôt trouver sa cause dans une pure subjectivité inconditionnée des systèmes cognitifs. C'est que, pour la sémantique cognitive, l'interaction entre le sujet et l'objet n'a pas d'histoire. Elle nous est immédiatement livrée avec ses propriétés définitives.

Ainsi, au moment décisif, la sémantique cognitive renonce à rendre raison de la métaphore et, par voie de conséquence, à éclairer la généalogie de nos concepts «abstraits ». Faute de prendre en compte la dimension historique du symbolisme, cette approche ne veut connaître que l'alternative entre une tentative de dissolution objectiviste du sens figuré, vouée selon elle à l'échec, et l'affirmation de son caractère quasiment irréductible, voire inconditionné. Ainsi s'explique la frustration répétée du lecteur de bonne volonté, persistant à chercher dans les pages de Lakoff, Johnson ou Sweetser autre chose qu'une glose sans cesse recommencée des métaphores étudiées. La vérité est qu'il n'existe pas à proprement parler pour nos auteurs d'explication possible. Tout comme le "prototype ", et pour les mêmes raisons, la référence à l'expérience humaine vaut pour eux comme un talisman qui les dispense d'expliquer les modalités précises ayant rendu possibles les projections métaphoriques.

\subsection{Réponse à des objections prévisibles : sur la place du linguistique et du culturel dans le système de Lakoff et Johnson}

$87 \mathrm{Au}$ fil de cet examen critique, nous avons souvent exprimé le regret que la sémantique cognitive ne prenne pas davantage en compte les dimensions culturelle, historique et linguistique dans sa théorie de la signification. Une telle objection pourra surprendre car nos auteurs manquent rarement une occasion d'évoquer ces dimensions de l'expérience humaine (en plus de sa dimension corporelle, sensori-motrice), lorsqu'ils évoquent la situation de l'homme dans le monde. On devrait donc s'attendre à ce que ces dimensions jouent pour eux un rôle non négligeable dans la compréhension humaine et, partant, dans leur théorie de la signification. Or, non seulement elles ne font pas l'objet de développements spécifiques, mais elles ne sont guère invoquées que pour servir de caution anthropologique à la théorie cognitiviste et enrichir l'argumentaire dressé contre la sémantique objectiviste - laquelle, on le sait, écarte par définition toute référence à la compréhension humaine du cœur de sa théorie de la signification. Si l'on observe avec attention la théorie et les études empiriques de Lakoff et Johnson, on constate en effet que ces dimensions se trouvent pour l'essentiel neutralisées comme telles et que leur poids spécifique est ignoré dans l'analyse de la signification linguistique et de la cognition humaine. Elles n'apparaissent guère en somme que comme des extensions, des réalisations particulières de la dimension préconceptuelle et corporelle de l'expérience humaine.

Ainsi Johnson peut toujours souligner (p. XII-XIII), pour infirmer le point de vue objectiviste - il renvoie également à ce propos au chapitre de Lakoff sur le relativisme que certaines langues non occidentales mettent en œuvre des systèmes conceptuels ${ }^{42}$ «fondamentalement différents du nôtre et même sans commune mesure avec lui ». Il ne songe plus en revanche à évoquer une telle disparité lorsqu'il entreprend de développer

Corela, HS-6 | 2007 
sa propre théorie sémantique. Tout porte même à croire qu'il serait le premier embarrassé si on lui rappelait ce point lorsque, pour parer à l'objection de relativisme que la sémantique objectiviste pourrait lui opposer, il souligne que les structures cognitives humaines, en tant qu'elles émergent d'une expérience identique dans ses grandes lignes d'un individu à l'autre (puisque fondamentalement corporelle), sont tout à fait compatibles avec une grande stabilité intersubjective. En réponse à Frege et aux objections objectivistes selon lesquelles «la reconnaissance du rôle central de l'imagination [telle que définie p. 194] dans la signification et la rationalité humaines conduisent à un relativisme où «tout se vaut » [anything goes] (p. 196), et pour écarter le spectre de la relativité des perceptions et des opinions, Johnson soutient en effet que « les schèmes d'images peuvent tout à fait avoir un caractère public, objectif (si l'on donne à objectif un sens acceptable) parce qu'ils sont des structures récurrentes de la compréhension humaine incarnée »(p.196). Il minimise donc ici les différences liées à l'histoire et à la culture.

Johnson reconnaît certes que les schèmes d'images ne sont pas nécessairement identiques pour tout le monde (p. 62) et il admet donc une certaine variabilité (résultant notamment des différences interindividuelles dans les compétences et l'expertise) concernant les gestalts expérientielles basiques sur lesquelles les individus fondent leur compréhension, mais il ajoute aussitôt que " nos corps sont très similaires eu égard à leur constitution physiologique ", ce qui justifie de penser que nous devrions «trouver des structures de gestalt communément partagées (si elles ne sont pas universelles) pour beaucoup de nos interactions physiques avec notre environnement » (ibid.). Ajoutons que, dans l'esprit de Johnson, une telle similarité d'ordre physiologique devrait suffire à assurer un partage de structures cognitives de niveau élevé, puisque les structures les plus complexes sont supposées dériver, par projection, de notre interaction corporelle avec le monde. Une certaine « objectivité » est ainsi rendue possible par la nature publique (cf. aussi p. 212), partagée, des structures profondes de notre compréhension humaine et la différence des systèmes conceptuels ne semble guère ici porter à conséquence.

On pourra donc parler d'un écrasement des dimensions culturelle et linguistique de l'expérience humaine au profit de sa dimension corporelle et physiologique (sensorimotrice) - plutôt que d'un système strictement unidimensionnel. On trouvera une confirmation de cet écrasement dans l'allusion suivante de Johnson au principe de la sélection naturelle (p. 208) :

Les concepts qui résultent de ces interactions [avec notre environnement] ont dû être, et continuent d'être, testés constamment instant par instant par des milliards de gens tout au long de notre histoire en tant qu'espèce. Ils ne fonctionnent pas trop mal ou alors nous ne serions pas ici pour en parler.

91 Tout suggère donc que, de l'avis de Johnson, l'élément déterminant pour la constitution de la cognition humaine telle que nous la connaissons réside dans l'interaction entre un homme fondamentalement invariant, défini avant tout, mais non exclusivement, par les conditions de son existence corporelle, et un environnement essentiellement naturel qui ne varie guère au fil du temps ni au gré de ses inscriptions géographiques. Le développement de la pensée humaine telle que nous la connaissons semble donc exclusivement, ou presque exclusivement, fonction de la sélection naturelle biologique ${ }^{43}$, régi par les seuls paramètres susceptibles d'affecter la survie de l'espèce. Ainsi, l'évolution des conditions socio-culturelles et linguistiques dans lesquelles se déploie l'expérience humaine ne semble jouer aucun rôle véritable dans le devenir de la cognition humaine tel qu'il est envisagé par Johnson ${ }^{44}$. Tout semble s'être joué dès les 
commencements de l'espèce (puisque l'impératif de survie a nécessairement joué dès ces premiers temps).

On soulignera qu'il existait d'emblée une certaine contradiction ou un certain tiraillement entre l'insistance sur la motivation sensori-motrice des significations humaines et la reconnaissance du rôle joué par le langage et la culture dans la conceptualisation. Ces deux influences ne peuvent en effet que se limiter mutuellement. Il faut choisir: ou bien le langage et/ou la culture ne font qu'opérer une sélection à l'intérieur d'un ensemble de schèmes préconceptuels préexistants ou disponibles a priori mais on ne peut dire alors qu'ils jouent un véritable rôle causal dans le fonctionnement de la pensée humaine ; ou bien ils ont réellement pour effet de modifier ou de reconfigurer les représentations issues des schèmes préconceptuels, mais la thèse de la motivation exclusivement sensori-motrice des significations humaines doit se trouver alors sérieusement amendée, complexifiée ou abandonnée.

93 Ainsi, pour le cognitivisme mentaliste, le linguistique et le culturel semblent bien se réduire à de simples manifestations d'un cognitif préconceptuel. Ils ne représentent guère pour lui que des relais, des "courroies de transmission ", d'un pur cognitif intramental, soustrait pour l'essentiel aux signes et à l'Histoire. La linguistique cognitive ne songe pas en effet que le langage humain pourrait changer en profondeur les propriétés et le fonctionnement de la cognition humaine. Pour nous au contraire, on l'aura compris, le niveau linguistique n'est nullement une annexe d'une cognition humaine intemporelle: il la reconfigure en permanence - en sorte qu'elle ne saurait plus apparaître seulement comme l'héritière d'une histoire évolutionnaire pré-humaine.

\section{Catégorisation et structuration des significations : des modèles cognitifs idéalisés à la théorie médiationniste}

Nous voudrions démontrer à présent, en réponse aux thèses de Lakoff et Johnson et de bien d'autres cognitivistes, qu'il est possible de définir un modèle non objectiviste des conditions nécessaires et suffisantes (CNS) aussi rigoureux et contraignant que le modèle traditionnel connu sous ce nom et cependant totalement immunisé contre les objections que la sémantique cognitive mentaliste lui a opposées.

Nous commencerons par montrer que, paradoxalement, la sémantique cognitive ellemême ne peut véritablement se passer des CNS. Puis nous argumenterons en faveur d'un modèle pluriel de la symbolisation linguistique distinguant clairement entre catégories prélinguistiques et significations linguistiques, ce qui nous amènera à marquer nos distances à l'égard du modèle de structuration catégorielle défendu par Lakoff et Johnson. Nous pourrons alors spécifier notre propre modèle d'emploi des signes linguistiques, un modèle des CNS révisées d'inspiration médiationniste ${ }^{45}$. Nous proposerons enfin, en guise d'illustration, quelques contre-analyses de cas que la sémantique cognitive estimait rebelles aux CNS traditionnelles. 


\subsection{De la difficulté pour la sémantique cognitive de se passer des CNS} actuelle $^{46}$ n'a pas su proposer de véritable solution de remplacement au modèle des CNS. Cette lacune est d'autant plus préoccupante que nos auteurs tiennent les significations pour de simples reflets de catégories mentales. Faute, en effet, d'une théorie de la catégorisation en état de marche, c'est leur théorie de la signification elle-même qui se trouve frappée d'indétermination. L'apport théorique de la sémantique cognitive actuelle à la question de la catégorisation se limite ainsi à une réflexion sur la structuration interne des catégories conceptuelles et, plus négativement, à des attaques systématiques, au demeurant particulièrement intéressantes et stimulantes, contre le modèle traditionnel des CNS.

Ce n'est pas tout. Il nous semble en effet que, sur ce thème de la catégorisation (entendue au sens étroit comme l'ensemble des mécanismes permettant de rattacher un faisceau de percepts à une catégorie), la sémantique cognitive a opéré discrètement un repli essentiel par rapport aux propositions provocantes de la version dite standard du prototype (pour reprendre la terminologie de Kleiber (1990). Ce repli ne se marque pas seulement à la réintégration, dans le modèle proposé, de catégories dites classiques, même si cette concession en a sans doute facilité beaucoup d'autres. Le modèle général de la structuration des catégories développé par la sémantique cognitive semble en effet présenter une différence notable d'ancrage expérientiel entre, d'une part, les catégories classiques, les catégories scalaires et les schèmes d'images et, d'autre part, les catégories résultant de l'application aux premières de divers mécanismes d'extension (projections métaphoriques, métonymiques, ressemblances de famille), puisque les structures directement signifiantes ne semblent pouvoir se recruter que parmi les premières. Or on voit mal comment nos sémanticiens, s'ils entreprenaient de se doter d'une théorie de la catégorisation en bonne et due forme, pourraient se dispenser de recourir à des conditions nécessaires et suffisantes (fussent-elles assouplies) pour les catégories à fort ancrage expérentiel, puisqu'aucun modèle n'est proposé en remplacement du modèle des CNS. Bien que la sémantique cognitive, encore une fois, ne se soit jamais souciée à notre connaissance d'apporter une réponse claire à cette question (celle de la catégorisation au sens strict), il est donc permis de tenter de spécifier le modèle positif de la catégorisation qui pourrait ou devrait être le sien moyennant de combler les lacunes de l'approche proposée à l'aide du seul modèle reconnu par la sémantique cognitive comme susceptible d'offir un principe adéquat, à savoir ( !) le modèle des CNS - le "prototype ", rappelonsle, n'étant considéré que comme un effet. Plus précisément, le modèle adopté devrait reposer, nous semble-t-il, sur la distinction de deux grands types de cas :

1. les cas où la catégorie considérée ne met en jeu aucun modèle «imaginatif » et est donc conforme aux CNS les plus traditionnelles (à cette nuance près que l'exigence de frontières nettes est suspendue). Le rattachement d'un exemplaire à ladite catégorie s'opère alors directement sur la base de l'examen des propriétés requises ;

2. les cas où la catégorie implique l'application d'au moins un modèle "imaginatif ». Le rattachement d'un exemplaire à une catégorie plurielle de ce type s'effectuera cette fois indirectement, sur la base du rattachement préalable de l'exemplaire considéré à l'une des sous-catégories constitutives de la catégorie ${ }^{47}$, comme, par exemple, s'agissant de la catégorie conceptuelle mère [mother], telle qu'analysée par Lakoff, à l'une des sous-catégories 
suivantes: mère biologique, mère adoptive, mère porteuse, mère donneuse, etc.. Bien que les auteurs ne le précisent pas, il semble que ce rattachement sous-catégoriel ne puisse qu'être subordonné lui-même à un ensemble de CNS (avec frontières rigides ou non), puisque, dans le cas contraire, l'appartenance catégorielle des exemplaires demeurerait indécidable.

Ainsi, le modèle ne semble échapper aux CNS au niveau catégoriel que pour les retrouver au niveau sous-catégoriel. En d'autres termes, la sémantique cognitive mentaliste n'est finalement rétive au modèle traditionnel que dans la mesure où elle estime que les polysèmes «reflètent" d'authentiques catégories, égales en dignité aux catégories «reflétées » par les unités monosémiques... Nous verrons bientôt ce qu'il convient de penser de cette dernière thèse.

\subsection{Logique et langage : pour répondre à d'autres objections prévisibles}

Peut-être nous objectera-t-on que nous outrepassons les règles de l'argumentation scientifique en nous permettant de combler les silences de la sémantique cognitive et qu'une théorie sémantique peut fort bien se passer d'une théorie de la catégorisation au sens strict du terme. Rappelons toutefois que la sémantique cognitive n'a cessé elle-même de souligner la nécessité, pour la théorie sémantique, d'« interpréter » les symboles du langage, c'est-à-dire de définir les modalités selon lesquelles ils entrent en relation avec la réalité - réalité pensée, en l'occurrence, comme une réalité d'expérience et non comme une réalité objective. Or, la question de la catégorisation relève à l'évidence de ces modalités.

Peut-être nous opposera-t-on également que l'usage des significations dans les langues naturelles n'a pas lieu de se soumettre à des exigences de type logique, le langage ordinaire ne se confondant pas avec le langage logique pas plus qu'avec celui de la science.

Nous répondrons en premier lieu qu'il n'appartient pas à la théorie sémantique d'ostraciser quelque type de langage que ce soit au prétexte qu'il ne serait pas représentatif du langage dans sa généralité et qu'une théorie sémantique digne de ce nom devrait être capable, moyennant d'inévitables ajustements, de s'appliquer aussi bien au langage ordinaire qu'au langage scientifique, et au langage logique en particulier - ce dernier n'étant qu'un canton, passablement internationalisé aujourd'hui, des langues naturelles.

Nous soulignerons ensuite que la différence entre le langage ordinaire (à supposer que la signification de cette expression soit absolument univoque) et le langage de la science n'est pas une différence de nature (i.e. une différence de fonctionnement irréductible), et que cette différence, si l'on s'en tient au plan logique, tient surtout ${ }^{48}$ à une différence dans les connaissances requises de l'interlocuteur. La distinction entre un langage ordinaire qui ne serait que localement soumis à la logique et un langage scientifique qui y serait tout entier livré nous semble procéder d'une méprise profonde sur la nature de la contrainte logique inhérente au langage, contrainte qu'il vaudrait mieux qualifier d'opératoire pour la laver de tout soupçon d'intelligence avec une puissance extérieure. Cette contrainte logique n'est pas en effet externe au langage, mais constitutive de son fonctionnement pour reprendre, en l'adaptant à un nouveau contexte, la terminologie de Searle ${ }^{49}$. Le langage poétique contemporain lui-même, qui joue si volontiers des contradictions logiques pour déjouer nos représentations, nous offre certainement le meilleur 
témoignage du caractère irréductible de cette contrainte opératoire puisqu'il ne cesse de la présupposer dans le moment même où il entreprend de la mettre en crise. On se demandera peut-être comment il est possible de transgresser une contrainte déclarée irréductible. C'est qu'il convient d'établir une distinction entre: (i) les contraintes linguistiques règlant l'usage des signes, contraintes relevant clairement de la compétence proprement linguistique qui nous occupe ici ; et (ii) la combinaison de ces signes relevant, elle, du discours et, donc, des compétences discursives et des intentions des sujets parlants. Le locuteur, si tel est son désir, peut toujours se permettre d'enfreindre la logique dans son discours, la contrainte linguistique n'en continuera pas moins de commander toute interprétation que l'on pourra avancer de ses propos. En d'autres termes, la contrainte opératoire qui règle l'usage des signes est constitutive au niveau linguistique, en ce qu'elle régit l'interprétation des unités (on ne peut pas l'ignorer dès lors qu'on connait la langue sous peine d'être compris pour ce que l'on a dit plutôt que pour ce que l'on a voulu dire ${ }^{50}$ ), mais le fait de respecter ou non ladite contrainte à l'échelle de la combinaison des unités reste l'affaire du régime de discours choisi.

103 Ajoutons qu'ignorer la dimension logique des langues, ce serait faire bon marché des dimensions "épistémique ", « intersubjective » et "praxique» du langage (cf. Nyckees $2006: 24$ et 39 et $2003: 66 \mathrm{sq}$.), autrement dit occulter le fait que les langues nous offrent des ressources d'une extrême sophistication pour penser le monde et agir ensemble. Ce serait oublier que, pour se comprendre entre eux et tirer du langage les infinies ressources qu'ils en tirent, tant pour analyser leurs situations et leurs expériences que pour agir conjointement selon des modalités extrêmement subtiles et variées, les locuteurs font montre d'une expertise particulièrement étendue qu'ils n'ont pu acquérir et conserver en mémoire que grâce à la maitrise de signes, linguistiques pour l'essentiel.

Une théorie de la signification doit donc être compatible avec les propriétés logiques du langage, c'est-à-dire, finalement, avec le fait que les langues, en définissant les conditions d'emploi des signes, rendent possible leur usage à des fins d'action et d'analyse du monde. Un tel point de vue n'implique ni de rejeter hors de la signifiance les régimes de discours non scientifiques ni de considérer que le monde qui nous entoure obéit à la logique - ce serait confondre le monde avec ses « représentations » linguistiques. Il faut admettre en revanche que tout usage de signes fonde des inférences et que des inférences contradictoires compromettent l'exploitabilité des messages - au moins dans le monde de l'expérience ordinaire et à moins, bien sûr, que le locuteur ou le récepteur ne prenne le soin de neutraliser les contradictions en différenciant les domaines d'application correspondant à chacun des signes utilisés.

L'approche cognitivo-mentaliste de la catégorisation se révèle pour sa part incapable de prendre en compte cette contrainte "logique » inhérente au langage. Elle n'est pas opératoire, comme l'a bien vu Kleiber (1990) ${ }^{51}$. Elle semble ainsi donner raison après coup à Frege qui, en son temps, fustigeait le psychologisme et estimait que l'on ne pourrait fonder de discours sémantico-logique rigoureux que sur une base objectiviste. Une théorie médiationniste, au contraire, qui traite le langage comme un ensemble de ressources intellectives et praxiques sans lesquelles la vie humaine telle qu'on peut la connaître serait impossible, ne saurait traiter à la légère les fondations linguistiques des raisonnements humains.

Il est vrai que les sémanticiens cognitivistes ne sont guère gênés par ce caractère non opératoire de leur approche parce qu'ils estiment que la catégorisation est une activité essentiellement individuelle et que les significations ne font que refléter les catégories 
qui en sont issues. La question de l'intercompréhension se trouve d'emblée éludée dans ce cadre naturaliste où il suffit que chaque sujet se comprenne lui-même pour que tous les locuteurs se comprennent entre eux. Pour un médiationniste au contraire, entrer dans le monde du langage, c'est précisément reconnaître autrui comme un alter ego, c'est-à-dire non pas comme un double, mais bien comme un autre je, dont l'histoire spécifique peut ne pas coïncider en tout point avec la sienne... En sorte que l'intercompréhension n'est jamais gagnée d'avance et que, avant même de prendre la parole, je dois d'une certaine manière me mettre à la place de mon interlocuteur et essayer d'anticiper, dans une exploration essentiellement intuitive, le sens qu'il pourra conférer à mes paroles et, pour commencer, aux signes mêmes que j'emploie.

Les sémanticiens cognitivistes, à vrai dire, semblent presque se réjouir, comme nous l'avons déjà montré, des défaillances de leur propre système d'explication, parce qu'ils entendent prouver qu'on ne peut rendre compte des catégories et de la signification en général sans prendre en considération la compréhension humaine. Ils croient trouver dans cette démonstration un argument imparable contre l'objectivisme, sans voir qu'elle les incite à une fuite en avant dans l'inexplicable et l'irrationnel. L'invocation de données irréductibles à l'analyse ne peut en effet tenir lieu d'explication, même dans le domaine des sciences cognitives... La référence à la boîte noire de la cognition individuelle ne dispense pas un cognitiviste digne de ce nom de proposer une authentique théorie de la catégorisation ni d'avancer une analyse plausible des processus susceptibles d'expliquer l'accord intersubjectif (relatif) sur les significations.

\subsection{Catégories conceptuelles et significations : une différence irréductible}

Afin de clarifier nos propres propositions, nous commencerons par discuter la thèse selon laquelle la catégorisation perceptive prélinguistique et la structuration de nos significations et de nos connaissances ressortiraient à une seule et même théorie de la catégorisation qui en ferait un ensemble de processus strictement mentaux, prélinguistiques et préconceptuels. Cette discussion nous amènera à rejeter l'assimilation mentaliste des significations, et des significations linguistiques en particulier, à des catégories conceptuelles ou préconceptuelles, et, par suite, à prendre nos distances par rapport au postulat d'une origine purement conceptuelle des significations linguistiques. Parler de catégories à propos des significations conduit en effet à ignorer ou à sousestimer l'originalité de l'économie des formes sémiologiques par rapport à celle des catégories prélinguistiques et à occulter ce que l'on peut appeler leur être pour la phrase et pour le discours ${ }^{52}$.

\subsubsection{La dimension intersubjective des significations}

Les formes sémiotiques représentent sans conteste, tout comme les catégories cognitives prélinguistiques en général, et les catégories perceptives en particulier, des ressources cognitives que chaque individu doit constituer pour son propre compte. Elles s'en distinguent cependant en ce que leur constitution ne se joue pas dans une relation duelle entre le sujet et le monde qui l'environne, mais dans une relation triangulaire ou triadique entre le sujet, le monde et le groupe (humain) auquel il appartient ${ }^{53}$. Si c'est toujours l'individu qui en dernier ressort "élabore" les significations, il ne le fait pas spontanément et à son seul usage, mais en s'efforçant de s'ajuster au mieux aux usages 
des groupes qu'il traverse, usages qu'il ne lui appartient pas de modifier de sa propre initiative. Cette propriété est bien sûr liée au fait que les formes sémiotiques à la disposition des locuteurs ne sont pas constituées au cours d'une seule vie d'homme et que le nouveau venu ne peut vraiment accéder aux expériences du groupe et interagir avec les autres membres de ce groupe qu'au prix d'un apprentissage relativement long. Ainsi, le « sens sémiotique » implique par définition un historique partagé avec des congénères (et plus précisément avec des "semblables ", autres sujets perçus comme tels). Il s'ensuit que la valeur d'un signe pour un locuteur est toujours fonction des valeurs qu'il croit avoir vu assigner aux occurrences antérieures de ce signe et que cette valeur n'est pas prévisible à partir des seules propriétés de la cognition individuelle.

Ajoutons que l'expression de catégorisation prélinguistique peut nous induire en erreur si on entend par cette expression la reconduction, par un sujet cognitif, d'un aspect (perçu) d'une situation donnée à un type, la catégorie, conservé en mémoire et accessible à volonté, hors contexte, sous la forme d'une image perceptive servant d'échantillon représentatif ou encore d'une liste de conditions à vérifier successivement. La catégorisation prélinguistique relève en effet pour nous, comme nous l'avons soutenu plus haut dans notre critique de la projection des schèmes d'images, d'un savoir-faire expérientiel inséparable de contextes déclencheurs. Il semble donc qu'en l'absence d'une langue ou d'un système symbolique, la "catégorie» reconstruite par l'observateur extérieur (le cognitiviste) ne soit pas accessible en tant que telle au sujet catégorisant. Tout au plus peut-on dire qu'un certain schème neuronal se trouve activé lors de la catégorisation. La catégorisation prélinguistique opérée par un sujet cognitif se résume donc pour l'essentiel, selon nous, à sa disposition à réagir de manière spécifique à divers aspects sensibles de son environnement extérieur ou intérieur. C'est pourquoi nous jugeons inadéquat d'en rendre compte par des listes de propriétés ou des faisceaux de CNS aussi bien que par des représentations mentales au sens fort du terme, étalons décontextualisés auxquels le sujet comparerait ses sensations lors des opérations perceptives. Le principe même d'une comparaison entre une sensation et une représentation interne mémorisée dans un format ou un autre nous parait engager la théorie cognitive dans une régression à l'infini puisque, pour pouvoir procéder à une telle comparaison, le sujet devrait avoir préalablement catégorisé sa représentation-étalon, ce qui, par définition, ne pourrait se faire sans comparaison. Pour chaque représentation à expliquer, la théorie cognitive se verrait donc obligée d'invoquer une représentation préalable et de présupposer à l'intérieur du sujet percevant un nouveau « sujet » percevant opérant la comparaison, et ainsi de suite à l'infini.

\subsubsection{La dimension syntactico-discursive des significations}

111 Une autre différence, fondamentale, entre catégories et significations linguistiques est que ces dernières sont toujours parties prenantes d'un discours et qu'elles comportent des instructions essentielles sur l'usage en phrase et en discours du signe auquel elles sont associées, instructions dont on ne peut les abstraire. Ainsi, même les catégories syntaxiques dites prédicatives ne se prêtent guère en français à un usage isolé en dehors des situations orales de «transmission » ou de dispositifs textuels tels que les titres ou les légendes de dictionnaires. Je peux, certes, dire "oiseau » à un très jeune enfant qui ne forme pas encore de phrases, en montrant un oiseau dans le ciel, mais il s'agit là d'un emploi métalinguistique très spécifique (présyntaxique) signifiant : « cela s'appelle oiseau » ou : «oiseau est le nom de ça»), emploi qui n'est d'ailleurs possible que pour des signes 
se prêtant à un usage ostensif. Dès que l'enfant aura dépassé ce stade d'apprentissage élémentaire, je recourrai nécessairement à une séquence incluant un déterminant (Un oiseau) pour commenter la même situation.

En traitant les significations linguistiques en termes de catégories, la linguistique cognitive tend donc à creuser un hiatus insurmontable entre unités lexicales (surtout de sens " concret $~_{54}$ ) et unités grammaticales, les premières étant censées entrer directement en contact avec le monde de l'expérience, à la différence des secondes. Les efforts de la grammaire cognitive pour remédier à cet écart qu'elle a elle-même créé ne parviennent pas à convaincre lorsqu'ils l'amènent à "resémantiser » artificiellement les unités grammaticales en les investissant de significations fondamentalement spatiales.

\subsubsection{Un modèle « pluri-historique » de la symbolisation linguistique}

113 Tandis que la sémantique cognitive assimile purement et simplement les significations linguistiques à des reflets de catégories cognitives pensées comme fondamentalement prélinguistiques, nous analysons pour notre part le phénomène de la symbolisation (ou de la signifiance) linguistique comme le produit dynamique de cinq types de processus historiques interconnectés correspondant à des temporalités distinctes ${ }^{55}$ :

\section{A) Processus relevant de l'histoire collective :}

1. les processus de catégorisation prélinguistique (I), antérieurs au développement du langage dans notre espèce et donc inaccessibles à l'observation directe ;

2. les processus de constitution historique des significations, processus d'élaboration des significations via l'histoire sémantique des langues (et, plus généralement, via l'histoire de l'ensemble des systèmes symboliques), processus qui eurent très tôt pour effet de sémiotiser de manière irréversible la catégorisation (initialement) prélinguistique en rendant nécessaire un apprentissage sémiotique spécifique ;

B) Processus relevant de l'histoire individuelle :

1. les processus de catégorisation prélinguistique (II), tels qu'ils se jouent, avant (voire au tout début de) l'acquisition du langage, dans l'histoire de chaque sujet humain ;

2. les processus d'acquisition individuelle des significations ;

3. la mise en ceuvre par les locuteurs des significations acquises, mise en oeuvre pouvant s'effectuer selon deux modes :

- 3.1. perception sémiotisée (et, plus généralement, analyse des situations informée par l'apprentissage linguistique);

- 3.2. usage de significations en discours.

114 Cette analyse appelle quelques précisions complémentaires :

115 - Soulignons d'abord que les catégories perceptives individuelles mises en œuvre par des êtres humains dotés de langage ne sont pas de fidèles représentants des catégories perceptives prélinguistiques puisque, depuis que l'homme est homme, elles n'ont cessé de se trouver reconfigurées par le langage (langues et systèmes symboliques).

116 - Les processus des niveaux A.2. et B.3. «bouclent » sans cesse :

- d'une part, au cours de l'histoire des langues, les significations sont en constante évolution, mais toujours à l'occasion de leur mise en œuvre par les sujets ;

- inversement, au cours de leur histoire propre, les sujets sont couramment exposés à de nouvelles significations et doivent être prêts en permanence à aménager leurs mondes de signification. 
117 - Les structures syntaxiques doivent être considérées comme des significations à part entière, associant un signifiant (un certain dispositif sensible) et un signifié (d'ordre instructionnel).

\subsubsection{Un modèle unique des conditions d'usage des signes}

118 Quel profit pouvons-nous tirer de ces analyses dans notre examen du modèle de structuration catégorielle avancé par Lakoff?

119 En premier lieu, lorsqu'il sera question de l'usage en discours des unités linguistiques, nous nous refuserons à parler de catégorisation et de catégories et nous parlerons respectivement d'application (ou d'usage) des signes et de significations. Cette précaution nous gardera de considérer les significations, à l'instar de Lakoff et Johnson, comme des réalités d'ordre strictement conceptuel. Ainsi, non seulement nos représentations concernant la signification des mots bachelor ou tuesday ont partie liée avec la culture, comme le note à juste titre Lakoff à propos de tuesday (p. 69), mais elles sont fondamentalement d'ordre linguistique, en ce sens qu'elles sont inséparables d'une certaine structuration de notre connaissance du monde qui s'est élaborée et continue de s'élaborer à la faveur du langage. Pas de semaine ni de mardi sans système sémiotique et, en l'occurrence, semble-t-il, sans une langue ${ }^{56}$. Il en irait de même a fortiori des structures calendaires extrêmement complexes adoptées par les Balinais et évoquées par Lakoff citant, à la même page, Clifford Geertz. De même, à l'évidence, pas de bachelor sans langage, car point de bachelor sans mariage et point de mariage sans langage ...

120 Nous soutiendrons ensuite, contre Lakoff et Johnson, qui invoquaient cinq types de structures catégorielles, l'idée d'un modèle unique des conditions d'usage des signes. Il nous faudra toutefois, pour justifier cette analyse, neutraliser d'une part la distinction d'ordre catégoriel établie par Lakoff entre catégories classiques et scalaires et réfuter d'autre part l'idée qu'il existerait d'authentiques catégories (complexes) métaphoriques, métonymiques ou radiales. Ou, pour le dire dans des termes moins suspects de mentalisme : nous commencerons par montrer que, sur le plan de l'application des signes, et quelle que soit par ailleurs sa validité sur le plan sémantique, la distinction entre significations gradables et significations non gradables ne se justifie pas et nous nous attacherons ensuite à réfuter l'idée qu'un polysème métaphorique, métonymique ou fondé sur une ressemblance de famille représenterait une signification unique, au même titre qu'une unité monosémique.

\subsubsection{Sur la distinction entre les significations gradables et les autres}

121 Précisons d'emblée que, dans notre modèle des CNS révisé, une signification n'a nul besoin d'avoir des « frontières [dénotationnelles] nettes » : il n'est aucunement nécessaire de pouvoir tracer a priori une limite bien précise, et la même d'un locuteur à l'autre, entre les exemplaires relevant de cette signification et ceux qui n'en relèvent pas. Il ne s'ensuit pas que la signification concernée doive être tenue pour floue. Soit, par exemple, les adjectifs gradables grand et petit évoqués par Lakoff ${ }^{57}$. Nous savons tous que, quel que soit le domaine d'expérience retenu, deux personnes peuvent diverger dans leurs appréciations concernant l'application de ces significations à certains exemplaires, et qu'une même personne pourrait même changer d'avis selon le moment où elle s'exprime. On remarquera cependant: $(i)$ qu'il est possible de proposer de ces adjectifs des définitions relativement indiscutables comme par exemple: «d'une taille supérieure / 
inférieure à la moyenne " pour la plupart des acceptions concrètes ; (ii) que le caractère apparemment «flou » de ces qualificatifs ne s'explique pas, contrairement à ce que l'on serait d'abord tenté de croire, par le fait que leurs significations respectives s'inscrivent sur une échelle de valeurs "continue» ou "gradable», admettant toutes sortes de degrés, puisqu'une mesure en degrés centigrades n'est pas réputée présenter un tel caractère. La vraie cause de cette impression de flou nous semble plutôt résider dans le sémantisme de type évaluatif inhérent à ces significations. Leur application à un objet quelconque implique en effet de la part du locuteur la mise en œuvre d'une norme, généralement implicite, appropriée à la classe à laquelle ce locuteur reconduit, implicitement ou explicitement, l'objet considéré ${ }^{58}$ : un objet donné n'est pas petit dans l'absolu mais eu égard à la norme retenue pour la classe à laquelle on le rattache. Le flou apparent de ces significations résulte donc exclusivement selon nous de la variabilité et de la pluralité des normes susceptibles d'être mobilisées par un sujet.

Ainsi, des significations comme celles de grand et de petit n'ont certes pas de limites absolument constantes ni d'un locuteur à l'autre, ni, pour un même locuteur, d'un instant à l'autre. On peut toutefois affirmer que, dans leurs acceptions courantes, chacun de ces termes signifie sensiblement la même chose pour tous les locuteurs du français. Au regard de ce critère, les significations de grand et de petit n'ont pas à proprement parler de frontières floues.

\subsubsection{La question de la polysémie}

Alors que la sémantique cognitive mentaliste postule l'existence de catégories conceptuelles métaphoriques, métonymiques et radiales, nous considérons pour notre part les polysèmes non pas comme des significations complexes, mais bien comme des ensembles de significations distinctes, entretenant il est vrai des relations perçues comme privilégiées par les locuteurs. La sémantique cognitive se croit obligée de traiter les polysèmes comme des catégories conceptuelles complexes parce que, par suite de la réduction des significations à des reflets de catégories conceptuelles individuelles, elle se rend incapable de considérer l'histoire sémantique, cette grande pourvoyeuse de démotivations et de remotivations, comme un ordre de causalité irréductible. L'histoire sémantique ne peut pour elle être explicative parce qu'elle ne fait à ses yeux que révéler les chemins suivis par une cognition éternellement identique à elle-même. La sémantique cognitive croit pouvoir se passer, pour cette raison, du niveau de la structure linguistique à laquelle elle ne reconnaît aucun statut causal et rapporter directement l'organisation sémantique des langues à une organisation conceptuelle dépendant des seules propriétés d'une cognition éternelle. Tout ensemble de significations associées à une même forme se doit en conséquence de représenter ipso facto une catégorie conceptuelle. En particulier, les significations figurées seront considérées comme devant participer de la même catégorie que les significations dites littérales.

On pourrait considérer que notre objection touchant au statut de catégorie conceptuelle accordé aux polysèmes n'est qu'une vaine querelle terminologique qui n'engage pas la théorie cognitive dans son ensemble. Il en va cependant tout autrement puisque Lakoff et Johnson considèrent bel et bien les catégories conceptuelles ainsi constituées comme des unités de la catégorisation au sens strict du mot. Ainsi, selon Lakoff, invoquant Austin et Wittgenstein :

les sens d'un mot peuvent être vus comme formant une catégorie, chaque sens étant un membre de cette catégorie. Du fait que les sens n'ont souvent pas de 
propriétés en commun, il n'y a pas de catégorie classique de sens que le mot pourrait désigner. Cependant, ceux-ci peuvent être vus comme formant une catégorie du type décrit par Wittgenstein. Il y a des sens centraux et des sens non centraux. Ces sens peuvent ne pas présenter de ressemblance (au sens d'un partage de propriétés), mais ils sont reliés l'un à l'autre par d'autres moyens que l'on peut spécifier. Ce sont de telles relations entre ces sens qui permettent de les considérer comme constituant une seule catégorie : les relations fournissent une explication de la raison pour laquelle un mot unique est utilisé pour exprimer ces sens particuliers. (Lakoff : 18)

Pour nous, au contraire, s'il est vrai que les polysèmes ont un statut particulier dans la pensée, en ce sens qu'ils obligent les locuteurs à appréhender avec une particulière attention les relations existant entre des significations isonymiques (partageant une même forme), il n'y a pas lieu de les considérer pour autant comme des catégories conceptuelles. A moins, bien sûr, qu'on ne décide d'entendre par concept, en un sens faible, l'aire sémantique associée à une forme unique... Mais tel n'est pas l'usage que Lakoff et Johnson font de ce mot. Catégorie conceptuelle doit s'entendre pour eux au sens strict, comme référant aux procédures de catégorisation. Or, il y a manifestement quelque contradiction à admettre comme relevant de la même catégorie des significations qui sont entre elles en relation dite métaphorique ou métonymique. On ne saurait dire, par exemple, que le mot clé définit une catégorie conceptuelle unique dont relèveraient à la fois ses occurrences en a) et en b) :

a) Donne-moi la clé du garage.

b) La clé de son succès, c'est son charisme.

Inutile de tirer prétexte ici de l'existence d'une forme unique pour postuler une catégorie conceptuelle unique sous-jacente aux deux emplois. Parler de catégorisation implique que l'on s'intéresse aux critères qui nous permettent de reconnaître ou de désigner quelque chose comme relevant de telle ou telle classe. Or personne ne trouverait normal que les mêmes critères d'identification ou d'usage puissent s'appliquer à la fois à ces deux occurrences du mot clé.

Ajoutons que l'existence de sens figurés en langue et en discours n'est pas de nature à poser de problèmes particuliers au modèle des CNS tel que nous le comprenons. Qu'une signification dite figurée soit perçue comme entretenant une relation privilégiée avec une signification dite littérale ( $c f$. à paraître b) n'empêche pas chacune de ces significations d'être associée à un ensemble spécifique de conditions. Une telle relation représente indubitablement une difficulté pour une conception objectiviste de la signification et des conditions nécessaires et suffisantes - puisqu'elle n'a d'existence que pour des sujets cognitifs. Elle n'en est pas une en revanche pour un modèle non objectiviste des CNS.

\subsection{Le modèle révisé des CNS et la théorie médiationniste des significations}

Il nous reste maintenant à préciser ce que nous entendons par CNS. Rappelons qu'elles ne concernent pour nous que les significations linguistiques, la catégorisation prélinguistique devant s'entendre comme le déclenchement individuel spontané de schèmes spécifiques d'activation neuronale. 


\subsubsection{Un modèle non objectiviste}

Défendre les CNS telles que nous les entendons n'implique nullement un objectivisme ${ }^{59}$, quelles qu'aient pu être, par le passé, les solidarités entre objectivisme et CNS et contrairement à ce que laisse entendre Lakoff, qui entretient une confusion systématique entre la thèse, ontologiquement neutre, $d u$ partage, qui voudrait que toute signification soit fondée sur des propriétés partagées et la thèse proprement objectiviste qui traite ces propriétés partagées comme des propriétés objectives, c'est-à-dire indépendantes de la compréhension humaine.

Il est vrai que, dans notre tradition dominée par le réalisme métaphysique, lequel affirme une isomorphie plus ou moins prononcée entre la structure de nos concepts et la structure du monde, le modèle des CNS fut le plus souvent interprété par défaut dans des termes qui semblaient devoir lier son destin à celui du réalisme, en ce sens que tout usage d'un concept ou d'une signification était censé requérir l'existence de propriétés objectives (au sens le plus fort de ce mot) communes aux exemplaires tombant sous ce concept ou cette signification ${ }^{60}$.

Pour nous, en revanche, les significations linguistiques ne sont ni des entités objectives ni des réalités purement subjectives. Ce sont des réalités “ interactionnelles”, produites par l'interaction entre l'homme et son environnement. Les CNS qui les spécifient ne doivent donc pas être entendues dans l'absolu comme des ensembles de propriétés objectives indépendantes de la compréhension humaine : elles sont fonction des connaissances et des croyances humaines et sont révisables en fonction de l'expérience. Ces CNS ne constituent même pas d'ailleurs des conditions nécessairement remplies par les segments d'expérience auxquels s'applique la signification. Ce sont seulement des conditions dont les locuteurs croient qu'elles sont effectivement remplies ou qu'ils choisissent en tout cas de considérer comme telles - s'ils ont lieu de penser par exemple que leurs interlocuteurs les tiennent pour effectivement remplies et qu'ils n'ont pas de raison sérieuse de les en dissuader. Les CNS n'en contraignent pas moins très sérieusement et très efficacement l'usage des expressions linguistiques.

Enfin, ces CNS révisées ne correspondent pas nécessairement à des propriétés (présumées) partagées par tous les exemplaires, puisqu'elles mettent souvent en jeu des «propriétés par défaut » et des " propriétés subsidiaires »- cf. ci-dessous notre analyse de mère. C'est dire que, tout comme Lakoff et Johnson, nous sommes ici redevables à Minsky et Putnam dont les cadres et les stéréotypes ont permis d'assouplir le modèle traditionnel des CNS en faisant leur place aux valeurs par défaut ${ }^{61}$.

\subsubsection{Des CNS relativisées}

3 Ces prémisses nous conduisent à redéfinir les conditions nécessaires et suffisantes en spécifiant le champ de leur nécessité et de leur suffisance. Nous le ferons en nous fondant sur l'intuition somme toute banale d'une corrélation systématique entre i) l'application, par un locuteur, dans un certain contexte, d'une certaine forme linguistique à un certain objet d'expérience et ii) et un certain ensemble de croyances que l'on peut en inférer chez le même locuteur relativement au même objet. Une telle corrélation ne doit donc pas être comprise comme l'effet de propriétés logiques indépendantes de la compréhension humaine; elle est de bout en bout relative aux sujets, ce qui ne condamne pas la 
sémantique au flou et à l'inexactitude, bien au contraire ( $c f$. à paraître a). C'est cette corrélation qui nous permet de parler de conditions nécessaires et suffisantes :

134 - Si, en effet, un locuteur applique le signe $x$ à quelque chose ${ }^{62}$ (i.e. le qualifie de $x$, le désigne ou, plus généralement, le traite ou le présente comme $x$, etc.), alors il est nécessaire qu'il admette ${ }^{63}$ que ce "quelque chose » satisfait un certain ensemble de conditions qu'il croit attachées à l'usage du signe $x$.

135 - Inversement, il suffit qu'un locuteur croie (ou feigne de croire) que quelque chose satisfait la totalité des conditions qui régissent l'usage de $x$ pour qu'il puisse appliquer le signe $x$ à ce « quelque chose ».

La "nécessité » et la «suffisance » résident donc exclusivement ici dans le rapport qui s'établit entre l'usage d'un certain signe et les croyances (effectives ou affectées) d'un locuteur concernant certaines données de l'expérience.

On notera qu'un tel modèle n'est nullement réservé aux significations et aux concepts du langage scientifique. Il s'applique tout aussi aisément aux significations du langage ordinaire. Il n'est pas non plus réservé aux significations dites « catégorématiques » ou prédicatives. Le raisonnement formulé ci-dessus nous paraît valoir en effet dans ses grandes lignes quelle que soit la catégorie syntaxique concernée. Les significations dites syncatégorématiques devront toutefois être considérées ici comme des significations au second (ou au nième degré), c'est-à-dire comme des ressources permettant de « recatégoriser » une expérience déjà sémiotisée, de "surdire » ou de "sous-dire » en somme une expérience déjà dite.

Ainsi, pour nous en tenir à un exemple assez rudimentaire, nous pouvons dire que la signification $^{64}$ de la suite déterminant défini $+N$ en français, où $N$ est un substantif éventuellement expansé consiste à présenter le référent de l'expression référentielle considérée comme déjà connu ou accessible par le destinataire.

\subsubsection{Une théorie casuelle de la signification}

Une des difficultés majeures de l'analyse sémantique tient au fait que cet ensemble particulier de conditions qui définit une signification ne peut être que partiellement explicité - il n'a d'ailleurs guère besoin de l'être dans l'usage quotidien du langage. Ces conditions d'emploi n'en sont pas moins à la fois immédiatement accessibles aux locuteurs et on ne peut plus impérieuses pour leur pratique. Non pas parce que leur mémorisation résulterait essentiellement d'impositions autoritaires ou de prescriptions extérieures, mais parce qu'elles se dégagent au contraire de l'usage lui-même et qu'elles se fixent dans la mémoire des locuteurs dans la mesure même où ils les identifient comme des ressources désormais indispensables à leur action dans le monde, à leur analyse de l'expérience ainsi qu'au « réglage » de leurs affects. C'est que les signes linguistiques ne représentent pas des moyens d'expression indifférents de concepts qu'ils se contenteraient de véhiculer; ils définissent les conditions mêmes de l'exercice de la pensée proprement humaine, laquelle opère grâce à eux et mobilise à travers eux l'expérience qu'ils ont enregistrée, mais qu'ils ont aussi permis de constituer et d'organiser. C'est en apprenant à signifier et à interpréter que l'on apprend les règles du jeu humain. de casuelle. Selon une telle théorie, la signification d'un signe $x$ pour un locuteur se définit 
comme la capacité qu'offre ce signe de présenter un certain aspect du monde comme une occurrence d'une classe ou d'un type dont un ou plusieurs autres aspects du monde ont été antérieurement reconnus ou constitués comme des occurrences (au sein du même groupe linguistique) du seul fait de se voir appliquer le signe $x$. Ainsi, pour un locuteur, appeler un aspect du monde chien, confiture ou Napoléon, c'est présenter cet aspect du monde comme reconductible à la même classe ou au même type que l'ensemble des occurrences qui, d'après ses souvenirs, ont été antérieurement qualifiées de chien, de confiture ou de Napoléon dans des conditions analogues au sein du même groupe linguistique. De même, utiliser en français la séquence le/la $x$, où $x$ représente un signe quelconque relevant de la classe $\mathrm{S}$ des substantifs (par exemple chien), c'est présenter un certain aspect du monde désigné à l'aide de ce signe $x$ comme reconductible à la même classe ou au même type que l'ensemble des aspects du monde qui, tout en ayant été désignés à l'aide de signes relevant également de $\mathrm{S}$, ont, de mémoire de locuteur, été présentés comme des cas de le/la $x$ dans des conditions perçues comme analogues ${ }^{65}$.

Il y a évidemment, dans cette analyse de la signification, une sorte de circularité qui se marque à la récurrence de la variable $x$ dans les différentes formulations que nous proposons et qui peut inspirer une vague impression de tautologie. Mais cette circularité n'est autre que la circularité même du langage, dont l'essence consiste, pour la théorie casuelle, en un renvoi toujours recommencé à d'autres usages. C'est à la faveur de ce renvoi incessant que se déduisent, se construisent et s'ajustent les notions et que s'opère une initiation au monde qui peut compter, comme on le sait, sur un nombre de connexions neuronales plus élevé que le nombre d'étoiles de l'univers.

\subsubsection{Les significations, « connaissances d'expert » multimodales}

Les CNS telles que nous les comprenons ne sont ni des définitions ni des descriptions. Elles ne constituent ni des listes de conditions analogues aux définitions classiques (celles des dictionnaires par exemple), ni des listes de traits qui devraient être vérifiés pour justifier l'usage du mot concerné. Elles ne sont pas même présentes d'une manière générale comme des conditions explicites dans la compétence des locuteurs. Rien n'impose de considérer en effet que toutes les conditions auxquelles est soumis l'usage des significations seraient explicites ou même explicitables. Ainsi, je peux distinguer sans la moindre difficulté un merle d'un corbeau tout en étant parfaitement incapable d'expliciter par des mots les différences qui les séparent. On n'en déduira pas que j'ignore la signification (littérale) des mots merle et corbeau. L'implicite est par définition inéliminable des processus sémantiques, le langage étant moins propre à décrire ou reconstituer un monde objectif qu'à baliser un espace commun et à y tracer des chemins ( cf. Nyckees 2001). D'abord, en effet, nous acquérons la plupart des significations linguistiques par recoupement et «en situation». Ensuite, toute mise en oeuvre d'une signification s'opère sur un fond d'implicite - sur le fond d'une expérience et de croyances supposées partagées. Ajoutons que les CNS n'ont que minoritairement et très partiellement un format verbal, même si l'expérience humaine dans son ensemble se trouve structurée par le langage.

Il reste à expliquer pourquoi la définition (verbale) d'un signe offre dans bien des cas (pour qui la comprend) un raccourci assez efficace et, somme toute, intuitivement acceptable de la signification de ce signe. La raison en est selon nous que la maittrise d'une signification représente une forme d'expertise. Les CNS telles que nous les comprenons constituent des ensembles de critères reliés entre eux, profondément engagés dans les 
expériences des sujets et la culture des groupes, organisés en circuits ou en réseaux pouvant atteindre une très haute complexité, et rendant les significations analogues, au plan de la structure et de la richesse informationnelle et expérientielle, à des « connaissances d'experts ». Comme ces dernières, elles émergent de l'exposition à un nombre ordinairement très élevé de situations; comme elles, elles sont complexes et difficiles à objectiver. Une telle complexité ne surprendra pas si l'on songe que l'acquisition du langage est un processus de longue durée se poursuivant tout au long de la vie des sujets et, à la lettre, un processus d'acculturation, opérant une (re)structuration continue de l'expérience et permettant l'apprentissage de savoir-faire multiples et complexes. Les CNS présentent toutefois avec les connaissances d'expert une différence majeure. C'est que le type d'expertise qu'elles révèlent se trouve le plus souvent partagé par un très grand nombre d'usagers. A cette compétence experte, si communément répandue dans le corps social, tout à la fois requise par l'exercice du langage et stockée dans les significations linguistiques, nous donnerons le nom d'expertence.

C'est cette expertence qui explique que, sans être en général explicitement présentes dans l'esprit des locuteurs, les CNS telles que nous les définissons n'en rendent pas moins possibles des raisonnements. Pour prendre un exemple simple, tout locuteur sait d'expérience, sans avoir jamais eu besoin d'y réfléchir, qu'un supermarché est un libreservice, dès lors qu'il sait d'expérience ce qu'est un supermarché et qu'il sait d'expérience ce qu'est un magasin en libre-service. Il sait, en particulier, que dans les deux cas il ne paie qu'arrivé aux caisses (sauf concession consentie par le supermarché à un commerçant dans son espace commercial), qu'il existe de grands et de petits magasins en libre-service et que tous les magasins en libre-service ne sont pas aussi grands et ne proposent pas une offre aussi diversifiée que les supermarchés. Ces éléments lui suffiront amplement pour répondre aisément à des questions telles que : tout magasin en libreservice est-il un supermarché ? ou : tout supermarché est-il un magasin en libre-service? Autrement dit pour saisir intuitivement ce qu'un logicien définira comme une relation entre espèce et genre et un sémanticien comme une relation d'hypo-hyperonymie.

\subsubsection{Des CNS révisables}

Dernier point essentiel : les CNS telles que nous les concevons sont révisables en fonction des expériences des groupes où elles ont cours. Comme nous l'avons vu, elles fonctionnent comme des normes internes fondant la possibilité d'une intercompréhension relative, d'analyses cohérentes de l'expérience et d'actions humaines efficaces et coordonnées. Ce rôle anthropologiquement, cognitivement et praxiquement structurant des CNS entraîne, lorsqu'elles sont prises en défaut, la mise en oeuvre de mécanismes régulateurs et de stratégies catégorielles permettant, selon les cas, de les sauvegarder ou de les remanier.

Des contradictions peuvent surgir, en particulier, entre notre définition de la catégorie et notre observation des exemplaires. Toute contradiction n'est certes pas automatiquement détectée. Mais lorsqu'elle l'est et qu'elle est perçue comme un obstacle récurrent par un ou plusieurs locuteurs, des procédures de sauvegarde ou de réparation catégorielle pourront être mises en oeuvre pour tenter de la surmonter ${ }^{66}$. 


\subsection{Quelques contre-analyses ${ }^{67}$}

147 Nous nous livrons depuis de nombreuses années à un exercice stimulant, consistant à tenter d'analyser en termes de CNS non objectivistes des significations que les sémanticiens cognitivistes estiment rebelles aux CNS traditionnelles. Nos propres réflexions sur les CNS sont, pour une grande part, le fruit de l'expérience que nous ont apportée ces tentatives réitérées.

Précisons que ces analyses ne sont pas destinées à offrir un reflet exact de la compétence des locuteurs, ne serait-ce que parce que leur format est exclusivement verbal. Elles visent plutôt, dans une perspective de "simulation", à offrir une sorte d'équivalent opératoire de la signification considérée à seule fin de démontrer que celle-ci n'est pas livrée au hasard ou auréolée de flou, comme le laisse trop souvent entendre la sémantique cognitive, et qu'elle exerce au contraire de puissantes contraintes sur l'usage que peuvent en faire les locuteurs

Nous avons toujours cherché en priorité à analyser les significations étudiées à l'aide de définitions de type classique. Mais nous avons peu à peu compris que si de telles analyses peuvent assez souvent convenir pour des significations passablement « abstraites » (cf.jeu ), il en va sans doute un peu différemment pour des significations plus « concrètes » (cf. oiseau) ou plus profondément ancrées dans la vie sociale ( $c f$. mère) - parce que les expertences qui leur correspondent peuvent se révéler extrêmement différentes d'un locuteur à l'autre ou que leur histoire sémantique est plus compliquée, etc. - ce qui peut conduire assez souvent à substituer des descriptions aux définitions.

\subsubsection{Oiseau}

150 C'est ainsi que la description de la signification littérale d'oiseau que nous avons nousmême proposée dans des travaux antérieurs nous parait aujourd'hui incomplète faute de prendre suffisamment en compte la variabilité des expertences concernant ces animaux ${ }^{68}$. Selon, en effet, que le locuteur aura ou non été « exposé » à tel ou tel des cas que l'on peut déclarer problématiques (kiwis, poules ${ }^{69}$, chauves-souris, exocets, écureuils volants, chevaux ailés, etc.) et selon qu'il aura eu conscience ou non des problèmes sémantiques qu'ils soulèvent (puisque tout locuteur n'est pas nécessairement amené à s'interroger sur la pertinence du choix du mot oiseau dans chacun de ces cas particuliers), la signification littérale précise qu'il assignera à ce terme pourra varier sensiblement du fait de la nature et du nombre des clauses suspensives susceptibles de sommeiller dans sa compétence lexicale et de s'activer si la situation l'exige. A moins, bien sûr, qu'il n'ait connaissance de la définition savante (zoologique) de ce mot, définition entièrement positive («l'oiseau se définit par la plume ») qui permet de trancher dans tous les cas connus. A défaut d'une telle information, il sera réduit à une entente strictement intuitive et essentiellement négative de cette signification dont une traduction en mots pourrait être : « animal volant non insecte » ou " animal volant non insecte ni chauve-souris », etc., la longueur de la chaîne définitoire pouvant considérablement varier selon l'étendue de ses connaissances et de ses croyances.

Une modélisation détaillée des faits concernés reste donc à proposer ${ }^{70}$. 


\subsubsection{Jeu} Wittgenstein opposant à cet égard échecs et marelle. Le divertissement, en effet, n'implique pas nécessairement le rire ni même le sourire. Il est vrai, d'autre part, que nombre de jeux présentent des enjeux, à commencer par les jeux d'argent ${ }^{71}$. Peut-être même tout jeu offre-t-il quelque chose à gagner, qu'il s'agisse d'avantages matériels ou de satisfactions symboliques comme la reconnaissance d'autrui ou le sentiment de pouvoir se fier à sa bonne étoile. La présence d'un enjeu n'affecte toutefois en aucune façon le caractère divertissant $\mathrm{du}$ jeu, pour autant bien sûr que l'activité concernée mérite vraiment ce nom. Si l'enjeu joue un rôle dans tout jeu véritable, c'est en effet un rôle subordonné, en ce sens que la fonction de divertissement y est toujours reconnue ou présentée comme la plus importante. Si, par exemple, le rapport hiérarchique entre enjeu et divertissement vient à s'inverser, on ne parlera plus de jeu. Ainsi, si quelqu'un triche ou qu'il se renfrogne parce qu'il a perdu, il sera traité de "mauvais joueur». On lui tiendra rigueur de ne pas «jouer le jeu». Par une fiction sociale commode, le premier enjeu du jeu n'est autre en somme que le jeu lui-même.

\subsubsection{Mère}

Nous avons montré dans une étude inédite qu'il était possible, contrairement à ce qu'affirme Lakoff (p. 74 sq.), de rendre compte, dans les termes de notre modèle des CNS 
renouvelées, de la signification littérale ${ }^{72}$ du mot mère $e^{73}$ ou, plus exactement, de l'aire sémantique de ce mot relative à la maternité dans le monde humain ${ }^{74}$ - une fois opéré un nécessaire «toilettage » des données fournies par Lakoff, puisque ce dernier, sans s'en expliquer, ne craint pas de traiter sur un pied d'égalité le mot mother et divers composés, plus ou moins figés, plus ou moins «compositionnels» (une step mother est-elle une mère ?...), formés à l'aide de ce mot.

La signification du mot mère ainsi entendue, d'une indéniable complexité, nous parait assez exemplaire puisqu'elle nous semble mettre en œuvre, s'agissant au moins des sociétés humaines, une valeur par défaut et une valeur subsidiaire. On ne parlera pas pour autant de polysémie car tout indique que, dans le genre humain tel que nous le connaissons, l'état de mère représente un statut (social et/ou affectif) générique et infrangible, neutralisant la diversité des situations effectives. La description que nous proposerons correspond donc à une signification unique et non pas à un «cluster » de significations ou de "sous-catégories conceptuelles" reliées entre elles comme le voudrait Lakoff.

Nous devrons distinguer néanmoins deux moments successifs dans l'histoire récente de ce mot.

Avant le développement des techniques médicales modernes, la signification de mère [humaine] pouvait se décrire de la manière suivante :

- Valeur par défaut: la mère (de $\mathrm{x}$ ) : est normalement (a) la femme qui donne (a donné/ donnera) naissance (à $\mathrm{x}$ ) et qui, de ce fait, (b) en vertu d'un principe très général, assure (a assuré/assurera) également son «éducation maternelle » (la part de l'éducation attribuée à la femme dans un couple de parents).

- Valeur subsidiaire : (c) Toutefois, si, pour une raison ou une autre, la « mère » ainsi définie ne peut plus ou ne veut plus assurer l'éducation de cet enfant, et si ce rôle est assuré par une autre femme, le mot de mère est alors appliqué normalement ${ }^{75}$ à cette dernière, et non pas à la première, qu'on appelle en ce cas sa "mère biologique " pour les distinguer l'une de l'autre (nous distinguerons pour notre part "mère biologique " en ce sens et «mère selon la biologie »).

La situation s'est sensiblement compliquée de nos jours par suite du développement des techniques médicales modernes de transplantation d'embryons et de fécondation in vitro. La femme qui, autrefois, accouchait d'un enfant était a fortiori celle qui l'avait conçu. Il n'en va plus de même aujourd'hui. Si le schéma à double détente présenté ci-dessus reste valable dans ses grandes lignes, il doit donc être notablement affiné. En bref, la signification du mot mère est aujourd'hui sujette à variation selon l'état du droit dans le groupe linguistique considéré (on pourra comparer à cet égard les législations française et américaine), mais aussi selon les valeurs propres, explicites ou implicites, réfléchies ou non, des locuteurs. La question actuellement en débat est en effet de savoir s'il faut réserver le titre de mère (dans sa valeur par défaut, c'est-à-dire, dans notre terminologie, le prédicat de "mère selon la biologie ») à la femme qui accouche de l'enfant (comme l'exige encore le droit français) ou à celle qui l'a conçu (comme le prévoit le droit américain), autrement dit s'il faut considérer que la femme qui donne naissance à un enfant est a priori celle qui accouche de cet enfant ou celle qui l'a conçu ${ }^{76}$. 


\subsubsection{Un sujet grammatical prototypique?}

\section{au cours de l'histoire individuelle - tout comme elle méconnait d'ordinaire le jeu des causes historiques -, l'approche mentaliste apporte une nouvelle preuve de son inaptitude à prendre en compte le dynamisme des phénomènes linguistiques et s'interdit à la fois d'en proposer une description adéquate et une explication convaincante. \\ 4. Epilogue : La double historicité du langage et de la cognition - Ou comment replacer la linguistique cognitive et les études de la cognition humaine sur leur axe véritable...}

données de l'acquisition, à prendre acte de la recomposition incessante des compétences

161 La sémantique cognitive de Lakoff et Johnson entend expliquer l'intégralité des phénomènes sémantiques en invoquant des mécanismes mentaux strictement individuels et, par voie de conséquence, sensiblement invariables à travers l'espace et le temps. Cette option mentaliste la conduit à ignorer, selon nous, deux aspects fondamentaux du langage et de la cognition propre à notre espèce : l'historicité des significations linguistiques, d'une part, et, d'autre part, ce que nous appelons la constitutivité sémiotique de la cognition humaine - son historicité sémiotique et, spécialement, linguistique. Toutes les critiques de la sémantique cognitive auxquelles nous nous sommes livré dans cet article n'avaient d'autre but que de manifester les conséquences dévastatrices de cette méconnaissance. Toutes nos contre-propositions et contre-analyses, symétriquement, procèdent du souci d'illustrer la fécondité théorique et empirique d'une approche prenant acte de ces réalités oubliées. 
Il nous reste cependant à préciser ce que nous entendons par ces formules abstraites, si nous ne voulons pas suggérer qu'elles n'expriment que de vagues intuitions.

\subsection{La constitutivité sémiotique de la cognition humaine}

Parler de constitutivité ou d'historicité (sémiotique) de la cognition humaine signifie en premier lieu que la cognition humaine a une histoire, ce qui revient à dire, d'une part, que ses propriétés ne sont pas invariables à travers le temps et l'espace, et, d'autre part, que le principe de son fonctionnement n'est pas renfermé dans les strictes limites biologiques des systèmes cognitifs individuels. Du fait, cependant, que la cognition demeure nécessairement nouée à des organismes spécifiques - même les livres n'existent en tant que tels que lorsqu'ils sont lus ou remémorés -, l'histoire de la cognition dans notre espèce se déploie elle-même selon une double temporalité :

- individuelle : à l'échelle de chaque vie humaine ;

- collective : à l'échelle de l'histoire des différents groupes et sous-groupes linguistiques.

L'articulation de ces deux temporalités s'opère à la faveur de la transmission intergénérationnelle, les individus se communiquant au fil des générations la mémoire d'expériences antérieures grâce à des «traces » sémiotiques ou fortement imprégnées de langage (signes de diverses natures, discours, gestuelle technique, artefacts, etc...).

Parler de constitutivité sémiotique (et, spécialement, linguistique) de la cognition humaine signifie en second lieu que la cognition humaine se trouve modifiée en profondeur par l'existence du langage :

- au plan collectif, d'une part, l'histoire de la cognition humaine, déposée dans des traces sémiotiques ou sémiotisées, récupérées et interprétées à travers les âges, est étroitement tributaire du langage et de l'histoire des significations sémiotico-linguistiques;

- au plan individuel, d'autre part, le développement cognitif est directement lié à l'acquisition du langage et, plus généralement, au développement sémiotico-linguistique, c'est-à-dire à l'initiation des individus aux ressources cognitives d'ordre sémiotique, et notamment linguistique, de leur groupe linguistique.

Si nous nous limitons au plan collectif, plus décisif pour notre démonstration puisque les processus qui en relèvent définissent l'horizon du développement individuel ${ }^{77}$, cette constitutivité sémiotique de la cognition humaine se vérifie essentiellement selon nous à trois niveaux. Du premier au troisième, le rôle du langage apparaît de plus en plus déterminant. Au premier et au deuxième, il nous semble relever de l'évidence. Son rôle de moteur de l'innovation cognitive, en revanche (au troisième niveau), paraîtra d'abord moins manifeste, mais il est certainement plus décisif encore pour notre compréhension du phénomène humain et il nous semble ouvrir ou rouvrir à la linguistique des perspectives trop souvent négligées.

\subsubsection{Le langage, vecteur de la transmission culturelle}

Les conditions de la vie et de la cognition humaines sont, comme on le sait, profondément changées par la culture. Or, le langage, et les langues naturelles en premier lieu, constitue sans conteste le vecteur nécessaire de la transmission culturelle intergénérationnelle. C'est d'abord par exposition au langage des aînés, mettant notamment en œuvre un certain lexique et une certaine morphosyntaxe, que les individus d'une population humaine accèdent aux savoir-faire techniques et à la culture de leur groupe. Par le 
langage, l'individu s'initie au dernier état du monde humain dans son groupe linguistique et, par lui, il en transmettra quelque chose à ses cadets, en partie volontairement, en partie à son insu. C'est cette transmission langagière qui joue évidemment le rôle fondamental dans ce qu'on appelle parfois l'évolution culturelle cumulative (cf. notamment à ce propos Tomasello 2004: 10 sq., 39 sq. et 156). C'est elle qui permet en effet, au fil du temps, la conservation des innovations dans la mémoire collective et, par voie de conséquence, leur multiplication, leur complexification et leurs hybridations, constat qui, soulignons-le, n'impose nullement d'adhérer à la thèse simpliste d'un progrès global linéaire de l'Humanité.

\subsubsection{Le langage, opérateur d'action conjointe (opérateur synergétique)}

De quelque manière qu'on trouve à l'expliquer, personne ne contestera le fait que l'existence du langage modifie en profondeur les conditions d'exercice de la pensée et de l'activité humaines en rendant possibles des formes de coordination et de coopération jusqu'alors inédites. Par le langage, les agents co-construisent un espace supra-individuel d'activité et de pensée. Ils se synchronisent à distance en élaborant des systèmes de coordonnées spatio-temporelles partagées, unifient leurs repères respectifs dans une perception globale transcendant les perspectives étroitement individuelles. Ils coordonnent leurs activités, se donnent des rendez-vous, des échéances, bâtissent des projets communs, nouent des pactes et des alliances. Dans ce monde d'action et de pensée conjointes, ils se savent parties-prenantes d'une archi-subjectivité en devenir, engagés dans une activité complexe d'ordre supra-individuel où ils s'inscrivent néanmoins comme des sujets à part entière, leur subjectivité se trouvant redéfinie par l'échange communicationnel et la réversibilité des rôles et des places. La division du travail et l'échange de biens et de services commencent ici. Une nouvelle économie de l'activité humaine s'impose, de manière apparemment irréversible.

On remarquera que ces solides évidences nous éloignent déjà sensiblement de l'univers individualiste, « présentiste » et adamique de la sémantique cognitive.

\subsubsection{L'histoire linguistique, moteur d'innovation cognitive}

170 Les processus que nous venons d'évoquer restent toutefois compatibles avec l'idée d'une complète ancillarité du langage réduisant sa fonction à la manifestation de potentialités inscrites depuis toujours dans les dispositions d'une cognition humaine d'ordre strictement individuel. Nous voudrions montrer à présent que le langage ne joue pas seulement un rôle instrumental, mais véritablement causal, via l'histoire des langues, dans l'évolution de la cognition humaine. Contrairement à certaines idées reçues, l'histoire linguistique constitue en effet selon nous une source d'authentiques innovations d'ordre conceptuel, c'est-à-dire, en réalité, d'innovations sémantiques. Ce rôle moteur du langage devrait nous permettre de mieux comprendre comment, à partir de différences cognitives initiales assez ténues, en apparence du moins, avec ses plus proches cousins (notamment dans la perception d'autrui, $c f$. Tomasello 2004 et 2005), différences elles-mêmes imputables à une évolution neurophysiologique, l'homme a pu dévélopper un monde de représentations et d'activités tout à fait spécifique.

171 Pour éclairer ces affirmations, prenons d'abord l'exemple d'une évolution sémantique d'ordre lexical que nous avons étudiée dans deux articles antérieurs ${ }^{78}$, celle des verbes et déverbaux latins qui, à partir de la notion d'un lien physique concret, ont développé la 
valeur d'obligation juridique - obligare, nectere et leurs dérivés, pour ne citer que les plus importants. Nous fondant sur un ensemble de données concordantes apportées par la «Loi des XII Tables » (milieu du IV siècle av. J.-C), témoignage irremplaçable sur le droit de la Rome archaïque, nous avons émis l'hypothèse - notablement enrichie depuis par une étude de Brachet (2005) -, selon laquelle c'est l'évolution même des pratiques sociales, à commencer par le développement d'une force civile de plus en plus efficace, qui a progressivement suscité l'émergence, pour chacun des mots de ce champ lexical, d'une signification plus "abstraite" irréductible à la première. Ce qui allait devenir l'obligation juridique est d'abord en effet une contrainte physique exercée par le créancier à l'encontre du débiteur en vue d'une réparation effective ou symbolique. L'enchaînement ou l'encordement du débiteur permet de se saisir de sa personne et d'en disposer à son gré ${ }^{79}$. Le développement de la force civile dans la cité antique conduisit peu à peu à abandonner ces pratiques, pénibles à des degrés divers pour les deux parties. On ne cessa pas pour autant de désigner les débiteurs comme des obligati, et la relation qu'ils entretenaient avec leurs créanciers comme une obligatio, probablement parce que l'évolution - et la généralisation des nouvelles pratiques - ne fut que progressive, mais, à coup sûr, parce que la menace de prise de corps planait sur les récalcitrants et que le souvenir des sanctions antérieures continuait à hanter les esprits. Dans ces conditions, le lien exprimé par obligare et par les termes de ce champ n'allait bientôt plus être perçu que sur le mode symbolique, l'enchaînement ou l'encordement du débiteur n'apparaissant plus que comme une mesure de dernière extrémité et, en tout état de cause, comme un cas particulier d'une situation générique plus abstraite, l'obligatio juridique. Celle-ci sera de plus en plus encadrée par les textes, et progressivement redéfinie, mais on peut encore la décrire à ce stade comme la contrainte exercée par la société à l'encontre des débiteurs, par tous moyens appropriés, en vue de la restitution des biens prêtés dans des délais et selon des modalités convenus.

Qu'on nous comprenne bien : la notion abstraite nouvelle n'existe tout simplement pas avant qu'obligare et ses synonymes, ainsi que leurs dérivés respectifs, ne se dégagent du sémantisme concret qui étaient initialement leur seul sens, c'est-à-dire, finalement, avant que la pratique du lien effectif ne se perde. Ce n'est donc que rétrospectivement, et parce que nous relisons les commencements à la lumière des aboutissements, que nous pouvons distinguer une valeur concrète et une valeur abstraite dans le premier sens d'obligare.

Mais cette notion abstraite n'est pas davantage le simple produit de la disparition du « lien » concret. Son émergence a requis en effet la conjonction de deux conditions :

1. l'existence d'un certain mot doté d'un certain sens ;

2. l'évolution sémantique de ce mot à la faveur d'un changement dans l'expérience.

174 En d'autres termes, il s'est produit un événement cognitif - l'émergence d'une notion nouvelle - à la faveur d'une variation sémantique, mais cette variation sémantique ellemême n'aurait pu se produire sans une continuité formelle, sans la présence d'un support signifiant stable, exactement de la même façon que les variations génétiques qui conditionnent l'évolution des espèces ne peuvent se produire qu'à la faveur d'une continuité dans le matériel biologique et non pas indépendamment de lui. La mutation sémantique procède donc d'une réplique avec variation, tout comme la mutation génétique. 
De manière analogue, l'évolution sémantique du mot latin pecunia, « richesse en bétail », puis « argent, fortune, richesse, monnaie », mot apparenté à pecus, « le bétail » (Ernout et Meillet ; cf. aussi Nyckees 1998a : 147) résulte selon nous de la conjonction de deux faits :

- une évolution socio-culturelle, d'une part: la lente évolution des modes d'échange économique - du troc à l'usage des monnaies métalliques en passant par des formes intermédiaires privilégiant certains supports tels que les têtes de bétail - avec, pour corollaire, déjà, le renouvellement des critères d'appréciation de la richesse ;

- la permanence, d'autre part, d'un mot susceptible d'enregistrer ces variations à la faveur de contextes verbaux appropriés.

176 Là non plus, le changement dans l'expérience n'aurait pas suffi. Sans l'existence d'un mot assurant la continuité d'un signifiant au-delà, et en dépit, des transformations de l'expérience, l'émergence d'une notion générique subsumant les diverses manifestations de la richesse et les diverses modalités de l'échange économique n'aurait pu avoir lieu.

C'est ici que se révèle toute l'importance, tant cognitive que linguistique, du phénomène de détachement sémantique, totalement ignoré par la sémantique cognitive, et qu'elle n'est malheureusement pas seule à méconnaître. L'analyse d'une évolution sémantique ne doit pas en effet se limiter à l'identification de circonstances déclenchantes, permettant d'isoler un « contexte de transition » (2006: $45 \mathrm{sq}$.) ; elle doit encore s'efforcer de définir aussi précisément que possible les modalités selon lesquelles la nouvelle signification s'est autonomisée, détachée de l'ancienne, soit pour la supplanter totalement, soit pour coexister avec elle (ibid. : 52). N'étudier que les transitions, négliger la différenciation des significations, n'avoir d'yeux en d'autres termes que pour la motivation, c'est ne faire que la moitié du chemin, c'est, une fois de plus, écraser le travail du temps historique.

Nous venons d'évoquer des évolutions d'ordre lexical, mais les évolutions sémantiques d'ordre morphologique et syntaxique sont tout aussi importantes, sinon davantage, pour éclairer la généalogie de la cognition humaine telle que nous la connaissons. La syntaxe et la morphologie ont leur histoire, qui mérite d'être retracée (et, dans les limites de la science, reconstruite), comme les théoriciens de la grammaticalisation, dans le sillage de Meillet, nous le rappellent aujourd'hui. Les comparatistes, pour leur part, sont depuis longtemps acquis à cette idée. Ils sont particulièrement bien placés pour savoir que les catégories syntaxiques ou les structures phrastiques qui nous paraissent les plus naturelles n'ont pas toujours existé. Ainsi, Bréal évoquait en son temps, en s'appuyant sur divers exemples, la lente genèse des catégories de l'adverbe, de la préposition, de la conjonction (1982[1897]:185 sq.); il formulait quelques conjectures concernant celles du substantif, de l'adjectif et du verbe, faute de disposer à leur sujet d'indices philologiques certains (191); il dérivait des démonstratifs les pronoms relatifs des langues indoeuropéennes (210sq.), etc. S'il n'était, certes, que trop enclin à les interpréter en termes de progrès de l'esprit humain, et de l'esprit occidental en particulier ${ }^{80}$, selon une tendance pour le moins fâcheuse de son époque, c'est à juste titre qu'il insistait sur ces "événements capitaux de l'histoire du langage ", lesquels ont indiscutablement leur équivalent - nul n'en disconviendrait aujourd'hui - dans l'histoire des autres familles de langue.

179 Du fait de ses postulats strictement mentalistes, la sémantique cognitive actuelle est très éloignée de pouvoir seulement imaginer une telle généalogie des significations linguistiques. Rapportant invariablement les phénomènes sémantiques à des processus et à une causalité de niveau strictement individuel, elle est conduite à postuler que les 
locuteurs d'une époque quelconque ont, en tant que sujet cognitifs humains, tous les éléments requis pour reconstituer adéquatement les mécanismes pouvant expliquer un changement de sens survenu à n'importe quelle époque. C'est pourquoi, ne guettant dans les évolutions du passé que la réplique de mécanismes mentaux éternels, elle ne peut que négliger les apports que pourraient lui fournir les sciences historiques, quand elle n'oublie pas purement et simplement les enseignements des plus grands diachroniciens du passé (cf. 1997a : 51-52).

Faute, d'autre part, de prendre la mesure du temps considérable qui a été nécessaire à la constitution des langues humaines telles que nous les connaissons - puisqu'elle croit pouvoir expliquer l'intégralité des phénomènes sémantiques par le jeu de mécanismes mentaux individuels, ne requérant ni médiation ni maturation particulière -, la sémantique cognitive se méprend du tout au tout sur les processus en œuvre dans le développement linguistique et cognitif individuel et ignore le caractère proprement initiatique de l'acquisition langagière.

Ainsi, sur les deux axes de l'historicité du langage, elle se prive systématiquement des ressources explicatives que pourrait lui apporter la prise en compte de la durée historique et se révèle incapable de donner un contenu autre que formel, et finalement abstrait, au concept d'expérience qu'elle ne cesse pourtant d'invoquer.

\subsection{La double historicité des significations linguistiques}

Qu'on ne se méprenne pas. Notre insistance sur l'historicité du langage n'implique en aucune façon qu'une linguistique cognitive médiationniste ne puisse être qu'historique au sens habituel du terme, c'est-à-dire glotto-diachronique, ni même qu'elle doive se consacrer exclusivement à la généalogie des significations humaines ou à l'étude de leur acquisition. L'éventail qui s'offre à elle est autrement plus large. Il convient en revanche d'insister sur la nécessité, pour une sémantique cognitive conséquente, de resituer toute signification (lexicale, morphologique ou syntaxique) sur un double système, individuel et collectif, de coordonnées historiques ${ }^{81}$. A chaque instant, en effet, toute signification est à la fois le produit d'une histoire collective multimillénaire et le résultat de l'histoire acquisitive d'individus particuliers qui ont dû se l'approprier et devront s'employer à la mettre à jour tout au long de leur vie. Il s'ensuit que, de près ou de loin et quelle que soit la perspective qu'elle se donne, toute étude linguistique est confrontée à la question de la transmission.

Si l'on admet que l'histoire des individus comme celle des groupes humains est, à chaque instant, le produit du rapport entre une certaine expérience linguistique et une certaine expérience du monde, on pourra distinguer quatre niveaux fondamentaux d'analyse pour une linguistique cognitive médiationniste, correspondant à autant de programmes complémentaires :

- et 2) les études onto- et glotto-diachroniques, d'une part, décrivent les évolutions des significations linguistiques (signes et structures) et s'efforcent d'identifier les événements et processus - situés à l'articulation des expériences mondaine et linguistique - dont ces évolutions sont les effets, dans l'ordre du développement individuel pour les premières, dans l'ordre de l'histoire du groupe pris comme référence pour les secondes;

- et 4) les études onto- et glotto-synchroniques, d'autre part, décrivent les états linguistiques qui résultent de ces événements et processus et s'efforcent de reconstituer : 
- pour les premières, les procédures mises en œuvre par les sujets à tel moment précis de leur développement linguistique, et, typiquement, de leur "acquisition", pour donner un certain sens aux unités (signes ou structures) linguistiques auxquelles ils sont exposés ;

- pour les secondes, les procédures mises en œuvre par les locuteurs ${ }^{82}$ d'un groupe linguistique ("communauté ", "groupe » ou " sous-groupe " ${ }^{83}$ ) pour donner, à un certain moment de l'histoire linguistique de ce groupe, un sens manifestement identique à certaines unités linguistiques ${ }^{84}$.

Résumons-nous. S'il est vrai que le langage ne requiert pas de la part des sujets humains une compétence linguistique innée, indépendante de la cognition générale - quelque grammaire universelle -, il ne s'ensuit pas qu'à elles seules les conditions biologiques individuelles de l'expérience humaine permettent de rendre compte du fonctionnement linguistique, contrairement au postulat le plus discret de la sémantique de Lakoff et Johnson. La preuve en est qu'une vie d'homme ne suffit pas à constituer les langues telles que nous les connaissons, ni au plan de la morphosyntaxe ni au plan du lexique. Il convient donc que la linguistique, et la sémantique en particulier, prenne acte de la double historicité des significations linguistiques, historicité indissolublement individuelle et collective. D'une part, en effet, l'acquisition linguistique est toujours acquisition d'un système déjà constitué et, de ce fait, est à la fois fonction de l'histoire des systèmes linguistiques et condition première de l'initiation des individus à une vie de société que le langage fonde et organise. D'autre part, l'évolution des systèmes linguistiques et des conceptualisations qui s'opèrent en leur sein met en œuvre des facteurs que la cognition individuelle ne saurait suffire à expliquer parce qu'ils procèdent de cette économie intersubjective originale de l'activité humaine que le langage a rendue possible. Ainsi, le langage est à la fois la première mémoire des groupes humains et la principale mémoire des individus, dépositaire de leurs perceptions, de leurs connaissances et de leurs savoir-faire essentiels.

\section{BIBLIOGRAPHIE}

BERRENDONNER, A., LE GUERN, M., PUECH, G., 1983, Principes de grammaire polylectale, Lyon, Presses Universitaires de Lyon.

BRACHET, J.-P., 2005, « Obligare et obnoxius : observations morphologiques et sémantiques », in Glotta, Zeitschrift für griechische und lateinische Sprache, M. Meier-Brügger, G. Meiser et H.-G. Nesselrath, vol. 81, Vandenhoeck \& Ruprecht.

BRÉAL, M., 1982 (1ère édition : 1897), Essai de sémantique. Paris, Gérard Montfort

BRONCKART, J.-P., 1996, Activité langagière, textes et discours : Pour un interactionnisme sociodiscursif, Lausanne-Paris, Delachaux et Niestlé.

CONSTANTIN DE CHANAY, H., et RÉMI-GIRAUD, S., 2002, « "Espèces d'espaces” : approche linguistique et sémiotique de la métaphore », in Mots, P. Bacot et S. Rémi-Giraud éds, $\mathrm{n}^{\circ} 68$ : 75-104.

ELIAS, N., 1996 [1984], Du Temps, Paris, Fayard 
ERNOUT, A. et MEILLET, A., 1985, Dictionnaire étymologique de la langue latine. Paris, Klincksieck., 4e édition.

GUIRAUD, P., 1986 (1967), Structures étymologiques du lexique français, Paris, Payot HOUDÉ, O. (dir), 2003, Vocabulaire des sciences cognitives, Paris, Quadrige / P.U.F. JOHNSON M.,

- 1987 : The Body in the Mind : The Bodily Basis of Meaning, Imagination and Reason, Cambridge, The University of Chicago Press..

- 1989, « Image-Schematic Bases of Meaning », Recherches sémiotiques / Semiotic Inquiry vol. 9 (1989) Nos. 1-2-3, pp. 109-118.

KERBRAT-ORECCHIONI, C., 1980, L'énonciation. De la subjectivité dans le langage, Paris, Armand Colin.

KLEIBER, G., 1990, La sémantique du prototype - Catégories et sens lexical, Paris, P.U.F.

LAKOFF G., 1987, Women, Fire and Dangerous Things. What Categories reveal about the Mind, Chicago and London, The University of Chicago Press.

LAKOFF G., et JOHNSON, M.,

- 1985 [1980], Les métaphores dans la vie quotidienne, Paris, Minuit.

- 1999, Philosophy in the Flesh : The Embodied Mind and Its Challenge to Western Thought, New York, Basic Books.

LAKOFF, G., et TURNER, M., 1989, More than Cool Reason : A Field Guide to Poetic Metaphor, Chicago, The University of Chicago Press.

NYCKEES, V.,

- 1994, « Sémantique, cognition, historicité - Quelques solutions aux problèmes posés par les théories du prototype ", in Scolia, $\mathrm{n}^{\circ} 1$ « Prototypes ", J.-E. Tyvaert éd., Strasbourg, PROPARLAN : 71-108.

- 1997a, « Pour une archéologie du sens figuré », in Langue française, n 113, P. Cadiot et B. Habert éd., Paris, Larousse : 49-65.

- 1997b, «Catégories sémantiques et historicité des significations », in Histoire, Épistémologie, Langage, t. XIX-1, Paris, SHESL et PUV : 97-119.

- 1998a, La Sémantique, Paris, Belin.

- 1998b, « Humaine référence : La sémantique cognitive face à l'objectivisme », in Sémiotiques, n

• 15, « Positions de la référence », Cl. Normand éd., Paris, Didier : 33-59.

- 1999, «Théorie sémantique et conceptualisation », in Sémiotiques, n 16, B. Pottier et V. Nyckees éds., Paris, Didier : 51-81.

- 2000a, La Théorie sémantique entre histoire, culture et cognition, synthèse présentée pour l'Habilitation à diriger des recherches, Université Marc Bloch, Strasbourg-II.

- 2000b, « Changement sémantique et déterminisme socio-culturel », in Mémoires de la Société de Linguistique de Paris, Nouvelle série, Tome IX, « Théories contemporaines du changement sémantique », J. François éd., Leuven, Peeters : 31-58. 
- 2000c, « La généalogie d'une signification abstraite : les mots de la ruse et de la tromperie en latin ", in Actes du XXIIe Congrès international de linguistique et philologie romanes, Bruxelles (23-29 juillet 1998), vol. VII, « Sens et fonctions », Niemeyer : 539-548.

- 2000d, «Quelle est la langue des métaphores? », in Cahiers de Praxématique, n 35, C. Détrie éd., « Sens figuré et figuration du monde », Montpellier, Presses de l'Université Paul Valéry : 115-139.

- 2001, « Description du monde, interaction et coordination ", in Par monts et par vaux, Itinéraires linguistiques et grammaticaux, Mélanges offerts au professeur Martin Riegel, C. Buridant, G. Kleiber et J.-C. Pellat éd., Louvain-Paris, Peeters : 286-304.

- 2003, «La perspective médiationniste en linguistique », in Variation, construction et instrumentation du sens, M. Siksou éd., Paris, Hermès : 47-72.

- 2005, « Qu'est-ce qu'une espèce ? », in Verbum, XXVI, n 1, « Polysémie et prototype », H. BatZeev Shyldkrot éd. : 7-17.

- 2006, « Rien n'est sans raison : les bases d'une théorie continuiste de l'évolution sémantique » in Aspects diachroniques du vocabulaire, D. Candel et F. Gaudin dir., Publications des Universités de Rouen et du Havre : 15-88.

- à paraître a) « Une linguistique sans langue ? Contribution à une réflexion sur les conditions d'émergence d'un sens commun », in Langages, « Linguistique et sens commun », G.-E. Sarfati éd.

- à paraître b) « Le sens figuré en langue et en discours : les sources linguistiques de l'énonciation métaphorique ", in Regards sur la métaphore - entre Orient et Occident, D. Struve et C. Sakai dir., Les Indes savantes.

PAVEAU, M.-A., 2006, Les prédiscours : Sens, mémoire, cognition, Paris, Presses de la Sorbonne Nouvelle

SCHAFF, A., 1970 [1964], Langage et connaissance, Paris, Seuil, Points.

SWEETSER, E.,1990, From Etymology to Pragmatics, Cambridge, Cambridge University Press. TOMASELLO, M., 2004 [1999], Aux orgines de la cognition humaine, Paris, Retz

TOMASELLO, M., CARPENTER, M., CALL, J., BEHNE, T., MOLL, H., 2005, « Understanding and sharing intentions : The origins of cultural cognition ", in Behavioral and Brain Sciences, $\mathrm{n}^{\circ} 28$, Cambridge University Press : 675-691.

VYGOTSKI, L., 1997, [1934], Pensée et langage, Paris, La Dispute.

WITTGENSTEIN, L., 1961, Tractatus logico-philosophicus suivi de Investigations philosophiques, Paris, Gallimard.

\section{NOTES}

1. Rappelons que l'objectivisme sémantique considère les significations comme des réalités objectives indépendantes de la compréhension humaine (cf. notamment Nyckees 1998b).

2. Ce que l'on peut, par conséquent, appeler le modèle de Lakoff et Johnson emprunte bien sûr à nombre de devanciers, célébrés et reconnus comme tels dans leurs ouvrages. Nous ne les citerons qu'exceptionnellement dans cet article pour ne pas allonger outre mesure notre contribution.

3. Le médiationnisme ainsi défini s'oppose d'une part au nominalisme, entendu comme l'ensemble des thèses identifiant purement et simplement la pensée humaine au langage ; et d'autre part à ce que nous appelons l'instrumentalisme linguistique, qui considère le langage comme la simple 
traduction d'une pensée qui lui préexisterait, à l'échelle de l'histoire individuelle comme à celle de l'histoire des groupes humains. Dans la perspective médiationniste, le langage est vu au contraire comme un ensemble de ressources intellectives et praxiques à la disposition des sujets ( cf. Nyckees 2003).

4. Cf. notamment Nyckees 2003, mais aussi 1998a, 1999, et 2000a, travaux dans lesquels, en conformité avec une certaine tradition philosophique, nous ne parlions encore que de conceptualisme linguistique. Nous préférons employer actuellement le néologisme de médiationnisme pour désigner cette conception en tant qu'elle définit (ou contribue à définir) un programme de recherche pour une discipline intéressée par la problématique de la cognition (psychologie du développement, sciences cognitives, linguistique, etc.) - sur ce point, $c f .2003$, p. $48 \mathrm{n} .2$.

5. Cf. notamment nos critiques de la sémantique cognitive et nos propositions alternatives dans : 1997a, 1998a, 2000b, 2000c, 2006, pour le changement sémantique ; 1994, 1997b, et surtout 1998a et 2005 , pour la question de la "catégorisation sémantique »; mais aussi, plus généralement, ou pour la critique de la théorie sémantique développée par la linguistique cognitive : 1998a et b, 2000a, 2000c ; enfin, pour un modèle médiationniste de la métaphore : $2000 \mathrm{~d}$ et à paraître b).

6. Sur ce point, $c f$. Nyckees à paraître a).

7. Cette présentation se situera sur un tout autre plan que celle esquissée dans notre article de 1998 b, où nous nous attachions essentiellement à présenter l'argumentaire dressé par nos auteurs contre la sémantique dite objectiviste et, tout particulièrement, contre la sémantique formelle.

8. Faute d'espace suffisant, nous n'évoquerons qu'incidemment la question du changement sémantique ( $c f$. à ce propos 1997a, 1998a, 2000c et, surtout, 2006).

9. Il s'agit ici du système qui produit nos concepts, plutôt que du système formé par nos concepts.

10. La sémantique cognitive prétend volontiers expliquer ou éclairer le langage à partir des propriétés générales de la cognition humaine, mais elle semble le plus souvent opérer en sens inverse et utiliser les données offertes par les langues pour argumenter en faveur d'une certaine théorie de la cognition ( $c f .$, par exemple, Lakoff : 113).

11. Toutes les citations sont traduites par nous.

12. Lakoff et Johnson (1999) prendront toutefois leur distance à l'égard de leur innéisme de la fin des années 80. Ils adopteront une position plus nuancée invitant à dépasser l'opposition de l'acquis et de l'inné en reprenant des arguments comparables à ceux d'Edelman.

13. On distinguera ici entre une interprétation forte (mentaliste) et une interprétation faible (médiationniste) du concept d'expression de la pensée par le langage (cf. Nyckees 2003: 50 sq).

14. Lakoff et Johnson reconnaissent leur dette à l'égard, notamment, de Berlin, Rosch, Hunn, Mervis, Tversky (cf. notamment Lakoff : 269).

15. Ce glissement a été bien observé par Kleiber (1990 : chap. 4).

16. Non seulement les modèles cognitifs sont censés être à la source des effets prototypiques, mais tous ces modèles (y compris les modèles propositionnels) sont, selon Lakoff, susceptibles de produire de tels effets.

17. S'il n'en continue pas moins à parler à longueur de pages d'effets prototypiques, c'est que le mot même de prototype - et le concept de «prototypicalité » - permet de fédérer un ensemble de cas invalidant les CNS. On ne peut donc pas véritablement parler d'une théorie du prototype chez Lakoff (1987), mais tout au plus d'une théorie des sources d'effets prototypiques.

18. Pour nos auteurs, et pour Lakoff en particulier, les effets prototypiques constituent dans la plupart des cas (on exceptera les "catégories classiques») une pierre dans le jardin de l'objectivisme en ce qu'ils obligent à prendre en compte la compréhension humaine.

19. Lakoff, p. 284, ajoute à cette liste un cinquième type de MCI, les modèles symboliques, qu'il avait définis p. 154 et qui n'ont qu'un statut théorique mineur. 
20. Rappelons qu'en psychologie cognitive, le format propositionnel de représentation des connaissances correspond à une « symbolisation abstraite de l'information, formalisable selon la logique des prédicats» («Propositionnel (format)», in Houdé (2003: 352) et exprimable en termes d'ensembles ou de réseaux comportant des nœuds et des liens, comme l'observe Lakoff, p. 69 .

21. « Un modèle propositionnel caractérisant notre connaissance au sujet du feu inclurait le fait que le feu est dangereux... » (Lakoff : 113).

22. Ces entités, propriétés et relations ne sont pas, insiste Lakoff, des choses du monde, mais des réalités conceptuelles.

23. Cf. supra les paragraphes consacrés aux projections métaphoriques et métonymiques.

24. Lakoff écarte clairement des structures radiales le cas où les extensions ne représentent que des instances spécialisées de la sous-catégorie centrale (p. 84). On pourra discuter d'un strict point de vue historique l'idée que les instances concernées seraient étendues par convention, puisque ces extensions sont évidemment le produit de l'histoire. En revanche, elles pourront, le cas échéant, être considérées comme telles par les locuteurs qui les utilisent, donc d'un point de vue synchronique.

25. $C f$. aussi, à propos du schème du CONTENANT : "Nous avons intimement conscience de nos corps comme de contenants tridimensionnels dans lesquelles nous mettons certaines choses (nourriture, air, eau) et d'où émergent d'autres choses (déchets de nourriture et d'eau, sang, etc.). D'emblée, nous faisons l'expérience d'une incorporation [containment] physique constante dans notre environnement (ces choses qui nous enveloppent)» (Johnson : 21).

26. Nous avons vu que Johnson avait conscience d'étendre le sens du mot métaphore au-delà de sa valeur habituelle. Cet abus de langage délibéré nous semble révélateur d'une tendance générale de la linguistique cognitive à minimiser le rôle du langage dans les opérations de pensée. A la stratégie plus ou moins consciente s'ajoute peut-être toutefois une confusion d'ordre logique. On peut craindre en effet qu'à force de travailler sur des énoncés censés traduire des réalités préconceptuelles, Johnson n'en soit venu à oublier que ceux-ci ne représentaient précisément que des traductions.

27. Il est vrai que la phrase suivante de Johnson, formulée à propos de la métaphore L'APPARENCE PHYSIQUE EST UNE FORCE PHYSIQUE, semble suggérer que toutes les projections métaphoriques ne se produiraient pas nécessairement à un niveau préconceptuel : « Beaucoup des connexions entre ces deux domaines [force physique d'un côté, apparence physique (corporelle) de l'autre] sont des projections expérientielles intervenant au niveau préconceptuel de notre compréhension » (p. 15, souligné par nous). Il reste que Johnson ne nous donne aucun détail sur les connexions qui se produiraient à un autre niveau.

28. On se rappellera que balance a, en anglais, un sens beaucoup plus concret que le mot français équilibre par lequel nous sommes obligé de le traduire.

29. Dans le même ordre d'idées, et contrairement à ce qu'estime Johnson (p. 7 sq.), on ne saurait affirmer que l'énoncé métaphorique «L'APPARENCE PHYSIQUE EST UNE FORCE PHYSIQUE » (supposé à juste titre sous-tendre l'ensemble des propos d'un locuteur cité p. 6) reflète purement et simplement une expérience non verbale ou préverbale, car toute verbalisation implique par elle-même une analyse de l'expérience. Ajoutons que cette métaphore constitue le nœud de l'argumentation d'un locuteur tentant de justifier, au cours d'un entretien, la tentation de viol qu'il éprouve en certaines occasions. Johnson, certes, ne s'intéresse pas ici à la dimension discursive de cette métaphore. Mais serait-il prêt à admettre que l'ordre des phénomènes argumentatifs est soluble dans le préconceptuel?

30. Il peut arriver également qu'une métonymie soit spécifique d'un mot - cas que nos auteurs n'évoquent pas, mais qu'ils admettraient sans nul doute -, l'important étant que des locuteurs perçoivent la relation entre ces significations comme une relation de « contiguité ». 
31. Ces modèles proprement «métonymiques" ne constituent donc pas des modèles cognitifs si l'on entend par là des modèles qui préexisteraient au langage : ils sont en effet inscrits en langue.

32. Le ton didactique adopté en général par Johnson ( $c f$. notamment p. 112 sq.), mais aussi par Lakoff, pour nous parler de ce que nous sommes censés ressentir spontanément face aux « concepts métaphoriques » a quelque chose d'assez troublant.

33. Il est, en l'occurrence, absolument illégitime de parler de "métaphore " puisque cette équation ne repose pas sur l'intuition d'une analogie. On remarquera en outre que la corrélation entre LE PLUS et le HAUT (MORE IS UP) n'est pas absolument constante : MORE, par exemple, n'est pas corrélé avec UP lorsqu'on verse un liquide sur une surface plane, dépourvue de bords ( $c f$. Constantin de Chanay et Rémi-Giraud, $2002: 81 \mathrm{n}$. 13) ; inversement, un UP abstrait (moral) n'est pas toujours corrélé avec un MORE, ni un DOWN abstrait (moral) avec un LESS. Et un UP concret (sensori-moteur) est souvent sans relation avec un MORE...

34. On ajoutera que, dans une synchronie particulière, nombre de métaphores discursives semblent instruites par des modèles déposés dans la mémoire linguistique et discursive des locuteurs, $c f$. Nyckees 2000d et à paraître b. Quoi qu'il en soit, Lakoff et Johnson, dans les ouvrages auxquels nous nous référons, ne s'intéressent à ces métaphores discursives qu'incidemment : en tant qu'elles sont censées se nourrir de « métaphores » cognitives.

35. Il reste à expliquer comment les sens abstraits de link et de ses équivalents ont pu se dissocier de leurs sens concrets et vivre d'une vie propre. Telle est la question fondamentale qu'il convient de se poser si l'on entend traiter d'un point de vue historique la relation entre le sens « littéral » et le sens figuré de lien, ou de link - mais c'est là précisément le type de question que la sémantique cognitive ne se pose jamais lorsqu'elle se penche sur des évolutions sémantiques ( $c f$. infra notre « épilogue »).

36. Cette distinction s'impose pourtant à tout le monde, et aux expérientialistes autant qu'à quiconque. Le moins objectiviste des théoriciens est en effet fondé à distinguer, sans se contredire, entre le chien et l'idée de chien... Il lui suffit de poser une fois pour toutes que nous n'avons jamais accès au «monde environnant » tel qu'il serait indépendamment de nous et que, par conséquent, le seul monde environnant dont nous puissions parler est toujours un monde perçu par un observateur - ce qui n'exclut pas que « quelque chose », un « réel » (ou, plutôt, du réel) se propose à son observation. Une telle position soulève donc beaucoup moins de problèmes logiques que la position objectiviste qui voudrait que l'on puisse connaître le monde tel qu'il existe indépendamment de nous...

37. Nous préférons parler ici d'aspect $d u$ monde pour ne pas postuler, à la manière des objectivistes, un monde prédécoupé - i.e. indépendamment de (et antérieurement à) tout sujet cognitif - en objets, propriétés, relations.

38. On se rappellera par exemple la description du schème d'image de la FORCE dont nous avons cité plus haut des extraits.

39. Nous définirons sommairement nos schèmes sensori-moteurs comme des dispositions à réagir de telle ou telle manière à tel ou tel ensemble de stimuli, dispositions non mobilisables hors contexte parce qu'impliquant des ensembles de couplages sensori-moteurs d'une haute complexité.

40. Nous interprétons ici librement la distinction entre know-how et know-what utilisée par Varela (2004, Quel savoir pour l'éthique?, Paris, La Découverte : 38-39), distinction empruntée à Hubert Dreyfus qui la reprenait lui-même de John Dewey.

41. Nous souscrivons pour notre part à la thèse d'une réductibilité - ou, plus exactement, d'une explicabilité - historique du «symbolisme» à partir d'un sensori-moteur chronologiquement premier. Pour des tentatives en ce sens, $c f$. nos analyses des mots latins de l'obligation juridique (1998, chap. 7 ; 2006 ; et, infra, 4.) et de la ruse (2000c).

42. On pourra regretter les connotations d'homogénéité et de monolithisme attachées à cette expression. 
43. On peut se réclamer de Darwin sans réduire la théorie de l'évolution à la seule question de la sélection des espèces biologiques.

44. Peut-être, dans le cas contraire, craindrait-il d'ouvrir une brèche à la thèse d'une inégalité entre les cultures ou à celle d'un progrès historique de l'humanité. Il n'est peut-être pas inutile de rappeler ici que, prise en elle-même, et contrairement aux versions qui ont pu en être proposées dans le cadre colonialiste, puis dans celui du racisme nazi, la théorie de l'évolution n'implique aucune espèce de jugement de valeur sur les types de réalisation de l'humain.

45. Notre analyse a sensiblement évolué depuis nos premiers travaux sur la question de la catégorisation (résumés aux chapitres 12 et, surtout, 13 de La Sémantique).

46. Nous ne parlons donc pas ici de ce que Kleiber a désigné sous le nom de version standard du modèle du prototype, version abandonnée assez tôt (selon Lakoff) par la sémantique cognitive.

47. Lakoff insiste volontiers sur le fait que l'appartenance d'une sous-catégorie à une catégorie doit très souvent s'apprendre au cas par cas - et n'est donc pas a priori prédictible.

48. La différence, sur ce plan, tient également à une différence de pondération entre les finalités intellectives (analyse de l'expérience) et praxiques (action avec ou sur autrui) assignées au langage par les intéressés - même si la fonction praxique n'est jamais éliminable et si son ordre d'efficacité ne fait que se déplacer dans le discours savant.

49. Sur le statut des «normes » linguistiques - normes internes, c'est-à-dire procédant des usages eux-mêmes et non pas de prescriptions ou de proscriptions extérieures (on fera toutefois la part des différents types de pression subies ou intériorisées par les locuteurs) -, cf. Nyckees 2006: 23 sq et plus particulièrement 32 à 42 .

50. A supposer bien sûr qu'il soit possible de dire à dessein autre chose que ce que l'on veut dire... 51. C'était déjà vrai de la version dite standard (cf. Kleiber 1990, Nyckees $1997 \mathrm{~b}$ et 1998a).

52. Il est vrai que, dans des travaux antérieurs, nous avons parlé nous-même des significations linguistiques comme de catégories d'un type particulier, faisant du mot de catégorie un terme générique subsumant significations linguistiques et catégories prélinguistiques tout en insistant à diverses reprises sur la différence de nature entre ces deux types de phénomènes. Nous éviterons désormais dans la mesure du possible de reconduire cette pratique terminologique pour écarter certains malentendus et mieux manifester la spécificité du linguistique et du sémiotique.

53. De caractère social, ou en tout cas collectif, à la différence des catégories prélinguistiques, les significations linguistiques répondent avant tout aux besoins d'une activité coordonnée entre agents.

54. Le concept de catégorisation accorde en effet une priorité ontologique au concret, à la différence de celui de signification. On ne dira guère, par exemple, que l'on catégorise quelque chose comme une sottise, comme de l'intelligence, comme sincère, comme beau, alors que l'on trouvera tout naturel de catégoriser quelque chose comme un chien ou comme du bleu.

55. Le modèle exposé ici présente quelques différences notables avec ses versions antérieures.

56. Les calendriers supposent également une objectivation artefactuelle ( $c f$. Elias 1996) : ce sont des outils sémiotiques concrets autant que des réalités linguistiques.

57. On pourrait développer sensiblement les mêmes analyses à propos de pauvre et de riche (Lakoff : 21) ou de bleu et de vert (Lakoff : 288).

58. Nous reformulons de cette manière la définition des adjectifs évaluatifs proposée par Kerbrat-Orecchioni (1980: 84-86).

59. Notre réévaluation du modèle des CNS a été parfois interprétée, à tort, comme une concession excessive à ce courant. De notre côté, nous n'avons peut-être pas toujours suffisamment marqué les différences séparant notre interprétation de ce modèle de sa version la plus courante.

60. La remise en question, par la psychologie cognitive puis par la linguistique cognitive, du modèle des CNS ainsi compris peut d'ailleurs s'analyser comme une retombée tardive des difficultés rencontrées tant par la méthode inductive en épistémologie des sciences que par le 
réalisme lui-même dans l'ordre de la réflexion métaphysique (notamment à travers l'odyssée exemplaire de la philosophie analytique).

61. Il convient selon nous d'appliquer au "stéréotype " lui-même les enseignements que l'on peut tirer du constat putnamien de la « division du travail linguistique » ou, plus généralement, de l'observation de la variabilité interindividuelle des significations linguistiques ( $c f$. Nyckees à paraître a ; et Berrendonner, Le Guern, Puech (1983). Le statut d'une valeur quelconque ne peut s'apprécier en effet qu'à locuteur constant. Ainsi, ce n'est guère que pour des personnes déjà un peu versées en zoologie et sachant qu'un tigre peut ne pas présenter de rayures que la propriété « avoir des rayures » pourra constituer une propriété par défaut de ces animaux. Pour la plupart des locuteurs, ignorant l'existence de tigres sans rayures, ladite propriété demeurera une propriété constante, à moins qu'ils ne soient informés de l'existence de mammifères albinos, cette information valant dérogation dans cette classe pour toute condition fondée sur la pigmentation.

62. On entendra ici par quelque chose " n'importe quel aspect de l'expérience ».

63. Le locuteur peut se rendre compte après coup qu'il s'est trompé concernant telle ou telle condition particulière (on ne mesure pas nécessairement toutes les conséquences de l'usage d'un signe, même lorsqu'on en maîtrise bien la signification).

64. Les études morphosyntaxiques synchroniques devraient consister, pour l'essentiel, à porter au jour les principes gouvernant les équivalences morphosyntaxiques mobilisées par les locuteurs.

65. Le signe à prendre en considération est ici la séquence le/la $x$ et non pas le seul élément le/la.

66. Nous avons nous-même amorcé une réflexion sur ces procédures dont nous avons brièvement rendu compte dans notre synthèse d'habilitation. Mais tout reste à faire.

67. Nous avons dû renoncer à intégrer ici une contre-analyse des classificateurs du dyirbal étudiés par Lakoff (1987) - classificateurs qui lui ont inspiré un titre d'ouvrage aussi original que discrètement provocateur. Nous ne reviendrons pas non plus sur notre examen critique d'une longue argumentation de Lakoff visant à démontrer que le concept biologique d'espèce serait rebelle à une analyse en termes de CNS (cf. 2005).

68. De même, nos premières analyses des lexèmes tasse, bol, chaise, fauteuil, tabouret ( $c f$. notamment 1998a : 312, sq.), qui se présentent sensiblement comme des esquisses de définitions, nous paraissent à présent un peu trop rapides même si nous les estimons justes dans leurs grandes lignes.

69. Pour ne rien dire des poussins et des oiseaux blessés ou malades et devenus de ce fait incapables de voler, cas particuliers faisant exception, comme les poules et les kiwis, au principe général selon lequel « tout oiseau peut voler ", mais pour une toute autre raison : en vertu d'une dérogation également générale concernant les individus jeunes, malades ou blessés.

70. Pour un début de réflexion à ce sujet, $c f .2000 \mathrm{a}$ (p.128 sq. ) et, sur la question de la variation, $c f$ . Berrendonner, Le Guern, Puech (1983) et Nyckees à paraître a.

71. Pour la question du sport et celle des jeux d'argent, cf. 1994, 1997b et 1998a.

72. Pour une défense-illustration et une rédéfinition des expressions de sens littéral et de sens figuré, cf. à paraître b.

73. Lakoff parlait bien sûr du mot mother, mais au regard de leur signification littérale, les problèmes posés par les mots mère et mother peuvent trouver, sinon une réponse exactement identique, du moins un traitement analogue dans ses grandes lignes.

74. Nous ne tenterons pas ici d'expliciter avec la même précision les conditions d'emploi du mot mère appliqué aux femelles du monde animal. Une telle analyse devrait s'attacher tout particulièrement au cas des mères nourricières prenant en charge la subsistance, la "formation » et la protection de petits qui ne sont pas les leurs. Les désigne-t-on néanmoins comme des mères? Les décalages éventuels dans les pratiques désignatives des locuteurs peuvent-ils être corrélés avec des différences dans les types d'activité ou d'interaction dans lequel ils sont engagés? Telles sont quelques-unes des questions qu'il conviendrait de se poser. 
75. Normalement, c'est-à-dire à moins d'une contestation de ce titre par un/des locuteur(s) ou à moins d'une référence manifeste, dans le contexte, au domaine biologique excluant toute prise en compte de la valeur subsidaire.

76. On remarquera que ce qui est ici en crise est moins l'unité de la signification de mère que cette signification elle-même, puisqu'il faut se garder de confondre polysémie et variation interindividuelle ou intergroupale (pour ne rien dire des conflits d'interprétation), la polysémie devant s'apprécier à locuteur (et à lecte) constant (cf. à paraître a).

77. Nous ne nous attarderons donc pas ici sur le rôle joué par l'acquisition du langage - et, plus généralement, par le développement linguistique - dans le développement cognitif de l'individu. Sur cette question, $c f$. en particulier Vygotski (1997), Schaff (1970) et Tomasello $2004: 157$ sq.

78. 1997a (repris dans 1998a) mais aussi $2006: 52-54$.

79. Jusqu'à la loi Poetelia Papiria (326), les créanciers garderont le droit d'emprisonner leur débiteur, de le vendre au-delà du Tibre, voire de le mettre à mort.

80. L'intention d'établir une hiérarchie des cultures, des groupes ou des « époques » linguistiques est tout à fait étrangère à l'approche médiationniste telle que nous la comprenons, qui n'a aucune raison de postuler que l'histoire serait ordonnée à la réalisation d'un type humain privilégié (sylvestre, bucolique, urbain, savant ou technicien). En l'absence de critères objectifs permettant de déterminer scientifiquement le poids relatif des diverses innovations et leur intérêt humain ou moral, les qualifications de progrès ou de régression n'ont pas leur place dans des travaux de sciences humaines ou de sciences cognitives et relèvent, dans le meilleur des cas, de l'éthique.

81. On laissera de côté ici la dimension de «l'historique » du cours d'interaction.

82. On s'intéressera essentiellement ici, pour des raisons d'ordre à la fois pratique et méthodologique, aux locuteurs adultes (ou adolescents).

83. Ce terme doit être entendu de manière récursive : un groupe est constitué de sous-groupes eux-mêmes constitués de sous-groupes, et ainsi de suite jusqu'à épuisement des communautés de sens concernant une ou plusieurs unités données.

84. Une étude (glotto)synchronique ne s'intéresse, par définition, qu'à des significations partagées à l'échelle d'un groupe linguistique quelconque dont les dimensions peuvent être extrêmement variables selon les cas. Il en résulte que la cellule de base de l'étude (glotto)synchronique ne peut être que la signification particulière partagée (ou le plus petit micro-système sémantique partagé) par un nombre quelconque de locuteurs. Il ne s'agit certes pas d'attendre de toute étude linguistique qu'elle affecte systématiquement chaque signification étudiée à un groupe défini de locuteurs. Il importe en revanche que le principe d'une telle affectation soit compatible avec l'analyse proposée. Manière, en somme, de déjouer certaines illusions récurrentes dont la langue est l'objet - celle, en particulier, d'un langage indépendant de ses locuteurs et de ses inscriptions dans des mémoires organiques et, éventuellement, externes - et contre les erreurs méthodologiques qui peuvent en découler ( $c f$. à paraître a).

\section{RÉSUMÉS}

Cet article vise à la fois à exposer les très sérieuses objections que soulèvent selon nous les thèses de la sémantique cognitive actuelle et à démontrer, point par point, la supériorité d'une approche médiationniste, i.e. prenant acte du rôle déterminant du langage dans le développement 
de la pensée humaine, tant au plan individuel qu'au plan collectif. Nous réfutons ainsi l'opinion, maintes fois exprimée dans les travaux de sémantique cognitive, selon laquelle la seule "alternative» à l'objectivisme serait un expérientialisme de type mentaliste. Après une présentation impartiale des principales propositions et des grands concepts de la sémantique de Lakoff et Johnson, nous procédons à leur évaluation méthodique et exposons systématiquement les contre-propositions et contre-analyses qu'inspire une approche médiationniste. Est proposée notamment une reformulation médiationniste et non objectiviste du modèle des conditions nécessaires et suffisantes.

The aim of this article is both to expose some very serious objections raised by the theses of current cognitive semantics and to demonstrate, point by point, the superiority of a mediationist approach, ie. acknowledging the determining role of language in the development of human thought, both at individual and collective levels. Thus I refute the opinion, so often expressed in the works of cognitive semantics, according to which the only "alternative" to objectivism is a mentalist type experientialism. After an impartial presentation of the main propositions and major concepts of the Lakoff's and Johnson's semantics, I proceed to methodically assess them and to systematically expose the counter-propositions and counter-analyses that a mediationist approach inspires. In particular, a mediationist and non-objectivist reformulation is proposed for the model of necessary and sufficient conditions.

\section{INDEX}

Mots-clés : théorie du langage, signification, cognition, sémantique cognitive, mentalisme, médiationnisme, catégorisation, polysémie

Keywords : theory of language, meaning, cognitive semantics, mentalism, mediationism, categorization, polysemy

\section{AUTEUR}

\section{VINCENT NYCKEES}

Université Paris 7 - Denis Diderot, UMR 7597 - HTL 